\title{
Output power of a quantum dot laser: Effects of excited states
}

\author{
Yuchang Wu, ${ }^{1, a)}$ Li Jiang, ${ }^{2, b)}$ and Levon V. Asryan ${ }^{2, c)}$ \\ ${ }^{1}$ China University of Mining and Technology, Xuzhou 221008, China \\ ${ }^{2}$ Virginia Polytechnic Institute and State University, Blacksburg, Virginia 24061, USA
}

(Received 17 August 2015; accepted 26 October 2015; published online 11 November 2015)

\begin{abstract}
A theory of operating characteristics of quantum dot (QD) lasers is discussed in the presence of excited states in QDs. We consider three possible situations for lasing: (i) ground-state lasing only; (ii) ground-state lasing at first and then the onset of also excited-state lasing with increasing injection current; (iii) excited-state lasing only. The following characteristics are studied: occupancies of the ground-state and excited-state in QDs, free carrier density in the optical confinement layer, threshold currents for ground- and excited-state lasing, densities of photons emitted via groundand excited-state stimulated transitions, output power, internal and external differential quantum efficiencies. Under the conditions of ground-state lasing only, the output power saturates with injection current. Under the conditions of both ground- and excited-state lasing, the output power of ground-state lasing remains pinned above the excited-state lasing threshold while the power of excited-state lasing increases. There is a kink in the light-current curve at the excited-state lasing threshold. The case of excited-state lasing only is qualitatively similar to that for single-state QDs- the role of ground-state transitions is simply reduced to increasing the threshold current. (C) 2015 AIP Publishing LLC. [http://dx.doi.org/10.1063/1.4935296]
\end{abstract}

\section{INTRODUCTION AND THEORETICAL MODEL}

Excited states of carriers confined in semiconductor quantum dots (QDs) significantly affect the operating characteristics of injection lasers based on them (see, e.g., Refs. 1-21). In this paper, we develop a theory of output optical power of QD lasers in the presence of such states. The details of our model are discussed in the following text.

(I) To describe the actual situation of indirect injection of carriers into QDs, our model includes the bulk optical confinement layer (OCL) and processes therein-the carriers are first injected from the cladding layers into the OCL and then captured into QDs (Fig. 1).

(II) The carrier capture from the OCL into QDs is noninstantaneous - this presents one of the key components of our model. To describe the capture into a QD, we use the capture cross-section. As discussed in Ref. 22, no capture time into a single QD can be properly introduced; instead, using the capture cross-section, the capture time into the entire ensemble of QDs can be introduced that thus depends on the surface density of QDs.

(III) The spontaneous radiative recombination rate outside QDs (i.e., in the OCL) is quadratic in the carrier density $n_{\mathrm{OCL}}$ there; nonradiative Auger recombination (which rate is cubic in $n_{\mathrm{OCL}}$ ) can also be easily included into our model. It is the superlinearity of recombination rate outside QDs, which, combined with noninstantaneous capture into QDs and intradot relaxation, causes (i) saturation of output power of groundstate lasing and (ii) sublinearity of output power of excitedstate lasing with increasing injection current. Hence we do not assume monomolecular (linear in $n_{\mathrm{OCL}}$ ) recombination rate

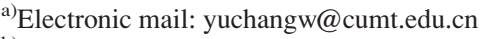

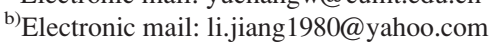

${ }^{c)}$ Electronic mail: asryan@vt.edu
}

outside QDs, i.e., constant recombination time outside QDs, which does not depend on $n_{\mathrm{OCL}}$. Monomolecular recombination outside QDs could be a factor only in the presence of high concentration of recombination centers there, which should be avoided in laser-quality structures. More importantly, even if such recombination is present, it will be first dominated by spontaneous radiative recombination and then Auger recombination with increasing injection current, i.e., with increasing $n_{\mathrm{OCL}}$. Monomolecular recombination outside QDs can cause neither saturation of output power of groundstate lasing nor sublinearity of power of excited-state lasing, both of which are important derivations from our model.

(IV) To mainly focus on the effects of excited-states, we assume that the carrier capture into and escape from the QD ground-state occur via the QD excited-state (Fig. 1). For the case of direct capture from the OCL into single-state QDs, the optical power was calculated in Refs. 22 and 23.

Depending on the parameters of the structure, there can be three possible situations for lasing. We consider them separately in the following text.

\section{GROUND-STATE LASING ONLY: HIGH GAIN FOR GROUND-STATE TRANSITIONS AND LOW GAIN FOR EXCITED-STATE TRANSITIONS}

If the maximum modal gain for ground-state transitions is higher than the mirror loss (the strict criterion will be formulated in the following text) and the maximum gain for excited-state transitions is lower than the mirror loss, the lasing will always occur via ground-state transitions.

\section{A. Rate equations}

Our model is based on the following set of rate equations: 


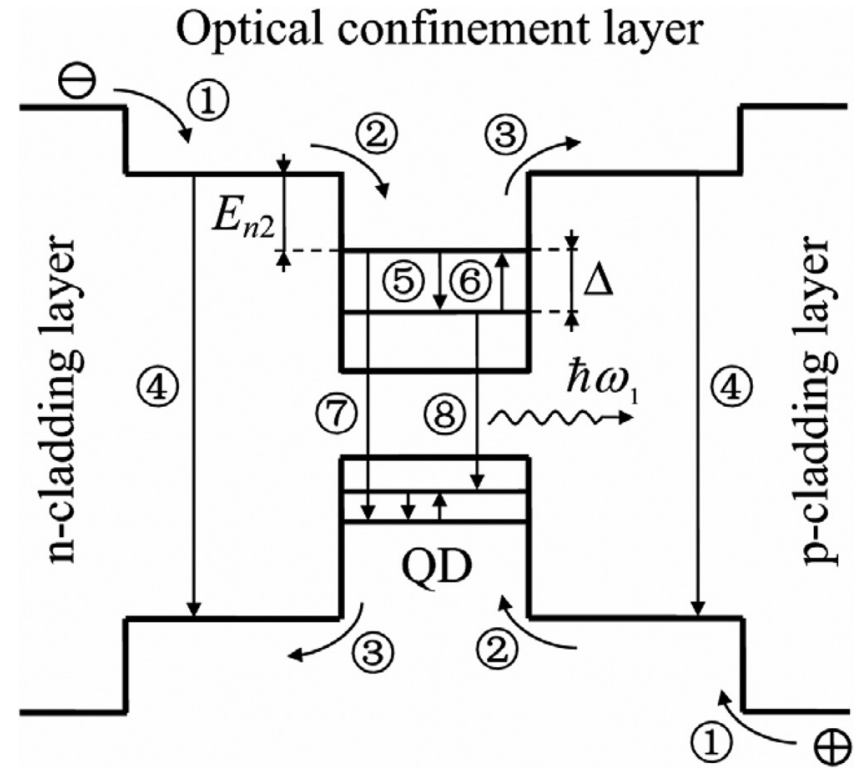

FIG. 1. Energy band diagram of a QD laser (the layers are not drawn to scale). The main processes (shown by arrows) are as follows: (1) carrier injection from the cladding layers to the OCL, (2) carrier capture from the OCL into the QD excited-state, (3) carrier escape from the QD excited-state to the OCL, (4) spontaneous radiative recombination in the OCL, (5) downward transition in QDs (intradot relaxation), (6) upward transition in QDs, (7) spontaneous and stimulated radiative recombinations via the excited-state in QDs, and (8) spontaneous and stimulated radiative recombinations via the ground-state in QDs.

for free carriers in the OCL

$$
\begin{aligned}
\frac{\partial n_{\mathrm{OCL}}}{\partial t}= & \sigma_{2} \mathrm{v}_{\mathrm{n}} n_{2} \frac{N_{\mathrm{S}}}{b} f_{2}-\sigma_{2} \mathrm{v}_{\mathrm{n}} n_{\mathrm{OCL}} \frac{N_{\mathrm{S}}}{b}\left(1-f_{2}\right) \\
& -B n_{\mathrm{OCL}}^{2}+\frac{j}{e b},
\end{aligned}
$$

for carriers confined in the excited state in QDs

$$
\begin{aligned}
\frac{\partial}{\partial t}\left(2 \frac{N_{\mathrm{S}}}{b} f_{2}\right)= & \sigma_{2} \mathrm{v}_{\mathrm{n}} \frac{N_{\mathrm{S}}}{b}\left(1-f_{2}\right) n_{\mathrm{OCL}}-\sigma_{2} \mathrm{v}_{\mathrm{n}} n_{2} \frac{N_{\mathrm{S}}}{b} f_{2} \\
& +\frac{N_{\mathrm{S}}}{b} \frac{f_{1}\left(1-f_{2}\right)}{\tau_{12}}-\frac{N_{\mathrm{S}}}{b} \frac{f_{2}\left(1-f_{1}\right)}{\tau_{21}}-\frac{N_{\mathrm{S}}}{b} \frac{f_{2}^{2}}{\tau_{\mathrm{QD} 2}}
\end{aligned}
$$

for carriers confined in the ground state in QDs

$$
\begin{aligned}
\frac{\partial}{\partial t}\left(2 \frac{N_{\mathrm{S}}}{b} f_{1}\right)= & \frac{N_{\mathrm{S}}}{b} \frac{f_{2}\left(1-f_{1}\right)}{\tau_{21}}-\frac{N_{\mathrm{S}}}{b} \frac{f_{1}\left(1-f_{2}\right)}{\tau_{12}}-\frac{N_{\mathrm{S}}}{b} \frac{f_{1}^{2}}{\tau_{\mathrm{QD} 1}} \\
& -\mathrm{v}_{\mathrm{g} 1} g_{1}^{\max }\left(2 f_{1}-1\right) n_{\mathrm{ph} 1},
\end{aligned}
$$

and for photons

$$
\frac{\partial n_{\mathrm{ph} 1}}{\partial t}=\mathrm{v}_{\mathrm{g} 1} g_{1}^{\max }\left(2 f_{1}-1\right) n_{\mathrm{ph} 1}-\mathrm{v}_{\mathrm{g} 1} \beta_{1} n_{\mathrm{ph} 1} .
$$

The physical quantities and terms entering into Eqs. (1)-(4) are presented in Tables I and II, respectively. We assume electron-hole symmetry in our model - that is why $n_{\mathrm{OCL}}^{2}, f_{2}^{2}, f_{1}^{2}$, and $\left(2 f_{1}-1\right)$ enter into Eqs. (1)-(4) instead of $n_{\mathrm{OCL}} p_{\mathrm{OCL}}, f_{\mathrm{n} 2} f_{\mathrm{p} 2}, f_{\mathrm{n} 1} f_{\mathrm{p} 1}$, and $\left(f_{\mathrm{n} 1}+f_{\mathrm{p} 1}-1\right)$, respectively.

Continuous-wave operation is considered here and

\begin{tabular}{|c|c|}
\hline$f_{1}$ & Occupancy of the ground-state in QDs \\
\hline$f_{2}$ & Occupancy of the excited-state in QDs \\
\hline$\tau_{\mathrm{QD1}}$ & Ground-state spontaneous radiative lifetime in QDs \\
\hline$\tau_{\mathrm{QD} 2}$ & Excited-state spontaneous radiative lifetime in QDs \\
\hline$\tau_{21}$ & Transition time from the excited- to ground-state in QDs (intradot relaxation time) \\
\hline$\tau_{12}$ & Transition time from the ground- to excited-state in QDs \\
\hline$v_{\mathrm{g} 1}$ & Group velocity of photons emitted via ground-state transitions in QDs \\
\hline$N_{\mathrm{S}}$ & Surface density of QDs \\
\hline$S=W L$ & Cross-section of the junction (QD layer area) \\
\hline$W$ & Lateral size of the device (QD layer width) \\
\hline$L$ & Cavity length \\
\hline$g_{1}^{\max }$ & Maximum modal gain for ground-state transitions in QDs \\
\hline$n_{\mathrm{ph} 1}$ & Density of photons (per unit OCL volume) emitted via ground-state transitions in QDs \\
\hline$\sigma_{2}$ & Cross-section of carrier capture from the OCL into the QD excited-state \\
\hline$v_{n}$ & Free carrier thermal velocity in the OCL \\
\hline$n_{\mathrm{OCL}}$ & Free carrier density in the OCL \\
\hline$b$ & OCL thickness \\
\hline$B$ & Radiative recombination constant for the OCL \\
\hline$j$ & Injection current density \\
\hline$\beta_{1}=(1 / L) \ln \left(1 / R_{1}\right)$ & Mirror loss coefficient for ground-state lasing \\
\hline$R_{1}$ & Facet reflectivity at the energy of ground-state transitions \\
\hline$n_{2}=N_{\mathrm{c}}^{\mathrm{OCL}} \exp \left(-E_{\mathrm{n} 2} / T\right)$ & \\
\hline$N_{\mathrm{c}}^{\mathrm{OCL}}=2\left(m_{\mathrm{c}}^{\mathrm{OCL}} T / 2 \pi \hbar^{2}\right)^{3 / 2}$ & Effective density of states in the OCL \\
\hline$E_{\mathrm{n} 2}$ & Carrier excitation energy from the QD excited-state to the OCL \\
\hline$m_{\mathrm{c}}^{\mathrm{OCL}}$ & Effective mass in the OCL \\
\hline$T$ & Temperature measured in units of energy \\
\hline$\hbar$ & Planck's constant \\
\hline
\end{tabular}
hence steady-state rate equations $(\partial / \partial t=0)$ are used.

Equation (3) can be written as follows at the steady state:

$$
\begin{aligned}
& \frac{N_{\mathrm{S}}}{b} \frac{f_{2}\left(1-f_{1}\right)}{\tau_{21}}-\frac{N_{\mathrm{S}}}{b} \frac{f_{1}\left(1-f_{2}\right)}{\tau_{12}} \\
& \quad=\frac{N_{\mathrm{S}}}{b} \frac{f_{1}^{2}}{\tau_{\mathrm{QD} 1}}+\mathrm{v}_{\mathrm{g} 1} g_{1}^{\max }\left(2 f_{1}-1\right) n_{\mathrm{ph} 1},
\end{aligned}
$$

TABLE I. Physical quantities entering into the rate equations (1)-(4). 
TABLE II. Rates of the processes entering into Equations (1)-(4).

\begin{tabular}{ll}
\hline \hline$\frac{N_{\mathrm{S}}}{b} \frac{f_{2}\left(1-f_{1}\right)}{\tau_{21}}$ & Downward transitions in QDs: Intradot relaxation \\
$\frac{N_{\mathrm{S}}}{b} \frac{f_{1}\left(1-f_{2}\right)}{\tau_{12}}$ & Upward transitions in QDs \\
$\frac{N_{\mathrm{S}}}{b} \frac{f_{1}^{2}}{\tau_{\mathrm{QD} 1}}$ & Spontaneous radiative recombination via the ground-state in QDs \\
$\mathrm{v}_{\mathrm{g} 1} g_{1}^{\max }\left(2 f_{1}-1\right) n_{\mathrm{ph} 1}$ & Stimulated radiative recombination (stimulated emission of photons) via the ground-state in QDs \\
$\sigma_{2} \mathrm{~V}_{\mathrm{n}} \frac{N_{\mathrm{S}}}{b}\left(1-f_{2}\right) n_{\mathrm{OCL}}$ & Capture from the OCL into the excited-state in QDs \\
$\sigma_{2} \mathrm{~V}_{\mathrm{n}} n_{2} \frac{N_{\mathrm{S}}}{b} f_{2}$ & Escape from the excited-state in QDs to the OCL \\
$\frac{N_{\mathrm{S}}}{b} \frac{f_{2}^{2}}{\tau_{\mathrm{QD} 2}}$ & Spontaneous radiative recombination via the excited-state in QDs \\
$B n_{\mathrm{OCL}}^{2}$ & Spontaneous radiative recombination in the OCL \\
$\frac{j}{e b}$ & Carrier injection to the OCL \\
$\mathrm{v}_{\mathrm{g} 1} \beta_{1} n_{\mathrm{ph} 1}$ & Mirror loss of photons \\
\hline \hline
\end{tabular}

which is simply the condition of equality of the net downward transitions rate in QDs (the left-hand side) to the net recombination rate via the ground-state in QDs (the right-hand side).

\section{B. Solutions of rate equations: Level occupancies in QDs, free-carrier density in the OCL, photon density, and output power}

Because $n_{\mathrm{ph} 1} \neq 0$, it follows immediately from Eq. (4) that the ground-state occupancy is pinned at its threshold value

$$
f_{1}=\frac{1}{2}\left(1+\frac{\beta_{1}}{g_{1}^{\max }}\right) \text {. }
$$

From Eq. (3), the excited-state occupancy can be expressed in terms of the photon density $n_{\mathrm{ph} 1}$

$$
f_{2}=\frac{1}{f_{1}+\left(1-f_{1}\right) \frac{\tau_{12}}{\tau_{21}}}\left[f_{1}+\tau_{12}\left(\frac{f_{1}^{2}}{\tau_{\mathrm{QD} 1}}+\frac{b}{N_{\mathrm{S}}} \frac{n_{\mathrm{ph} 1}}{\tau_{\mathrm{ph} 1}}\right)\right],
$$

where we introduced the lifetime in the cavity for photons emitted via ground-state transitions

$$
\tau_{\mathrm{ph} 1}=\frac{1}{\mathrm{v}_{\mathrm{g} 1} \beta_{1}}=\frac{1}{\mathrm{v}_{\mathrm{g} 1} g_{1}^{\max }\left(2 f_{1}-1\right)} .
$$

Using the detailed balance condition, the upward-todownward transition time ratio in QDs entering into Eq. (7) can be written as

$$
\frac{\tau_{12}}{\tau_{21}}=\exp \left(\frac{\Delta}{T}\right)
$$

where $\Delta$ is the separation between the energies of the excited and ground states in QDs (Fig. 1).

From Eq. (2), the free carrier density in the OCL is

$$
\begin{aligned}
n_{\mathrm{OCL}}= & n_{2} \frac{f_{2}}{1-f_{2}} \\
& +\frac{1}{\sigma_{2} \mathrm{v}_{\mathrm{n}}} \frac{1}{1-f_{2}}\left[\frac{f_{2}\left(1-f_{1}\right)}{\tau_{21}}-\frac{f_{1}\left(1-f_{2}\right)}{\tau_{12}}+\frac{f_{2}^{2}}{\tau_{\mathrm{QD} 2}}\right],
\end{aligned}
$$

or, taking into account Eqs. (5) and (6)

$$
n_{\mathrm{OCL}}=n_{2} \frac{f_{2}}{1-f_{2}}+\frac{1}{\sigma_{2} \mathrm{~V}_{\mathrm{n}}} \frac{1}{1-f_{2}}\left(\frac{f_{1}^{2}}{\tau_{\mathrm{QD} 1}}+\frac{f_{2}^{2}}{\tau_{\mathrm{QD} 2}}+\frac{b}{N_{\mathrm{S}}} \frac{n_{\mathrm{ph} 1}}{\tau_{\mathrm{ph} 1}}\right) .
$$

From Eqs. (1) and (2), the injection current density is

$$
j=e b B n_{\mathrm{OCL}}^{2}+e N_{\mathrm{S}}\left[\frac{f_{2}\left(1-f_{1}\right)}{\tau_{21}}-\frac{f_{1}\left(1-f_{2}\right)}{\tau_{12}}+\frac{f_{2}^{2}}{\tau_{\mathrm{QD} 2}}\right],
$$

or, taking into account Eqs. (5) and (6)

$$
j=e b B n_{\mathrm{OCL}}^{2}+e N_{\mathrm{S}} \frac{f_{1}^{2}}{\tau_{\mathrm{QD} 1}}+e N_{\mathrm{S}} \frac{f_{2}^{2}}{\tau_{\mathrm{QD} 2}}+e b \frac{n_{\mathrm{ph} 1}}{\tau_{\mathrm{ph} 1}} .
$$

Equation (13) simply states that the injection current goes into spontaneous recombination (via the OCL states and ground and excited states in QDs - the first, second, and third terms in the right-hand side, respectively) and stimulated recombination via the ground-state in QDs (the last term).

In Eq. (13), $f_{2}$ and $n_{\mathrm{OCL}}$ are functions of $n_{\mathrm{ph} 1}$ - see Eqs. (7) and (11). Using Eqs. (7) and (11) in (13), we obtain an expression for the injection current density as an explicit function $j\left(n_{\mathrm{ph} 1}\right)$ of the photon density. Our task is to calculate the inverse function, i.e., $n_{\mathrm{ph} 1}(j)$, and then the output power versus $j$. This can be done and a closed-form expression can be obtained from the solution of a quartic equation. This expression is, however, rather cumbersome and, for this reason, we use a different procedure to plot the functional dependences here. As shown in the preceding text, the quantities $f_{2}, n_{\mathrm{OCL}}$, and $j$ are expressed as explicit functions of $n_{\mathrm{ph} 1}$. Hence, we first consider $n_{\mathrm{ph} 1}$ as a variable, change it throughout the entire range of its possible values (from 0 to $n_{\mathrm{ph} 1}^{\max }$-see following text), calculate and plot $f_{2}, n_{\mathrm{OCL}}$, and $j$ versus $n_{\mathrm{ph} 1}$. The dependence of $n_{\mathrm{ph} 1}$ on $j$ is then simply obtained by switching between the abscissa and ordinate. Thus the light-current characteristic (LCC), i.e., the output optical power versus the injection current density, is calculated 


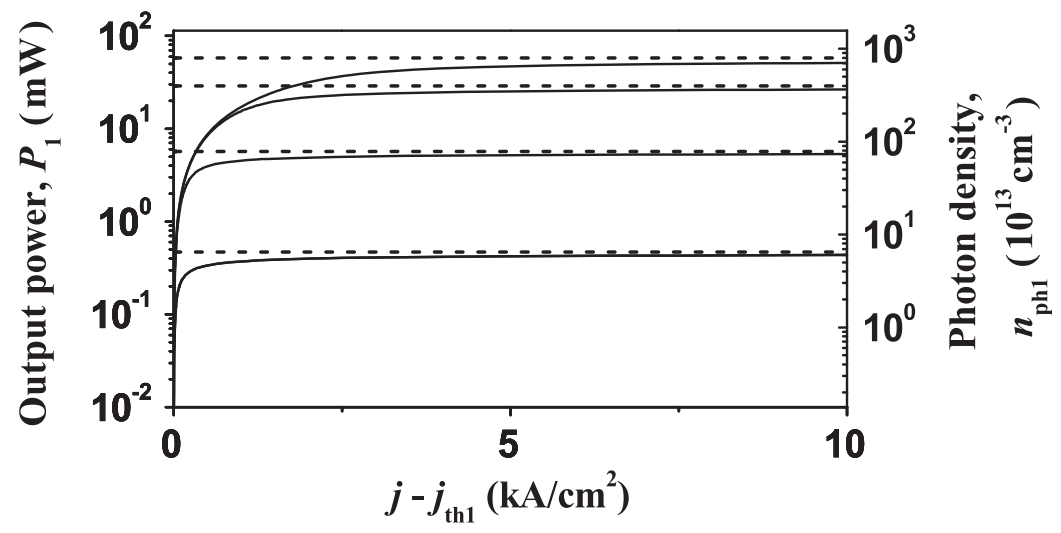

FIG. 2. Output power $P_{1}$ (left axis) and photon density $n_{\mathrm{ph} 1}$ (right axis) of ground-state lasing against excess injection current density. Both $P_{1}$ and $n_{\mathrm{ph} 1}$ go to zero at the lasing threshold $\left(j=j_{\mathrm{th} 1}\right)$; the lowest shown values of $P_{1}$ and $n_{\mathrm{ph} 1}$ are nonvanishing because the log-scale is used for the vertical axes. The horizontal short-dashed lines show $P_{1}^{\max }$ and $n_{\mathrm{ph} 1}^{\max }$ [Eqs. (21) and (20)]. A GaInAsP/InP heterostructure lasing near $1.55 \mu \mathrm{m}$ is considered here for illustration. ${ }^{34,35}$ In Figs. 2 and 4 , the parameters are as follows: $N_{\mathrm{S}}=6.11 \times 10^{10} \mathrm{~cm}^{-2}, \tau_{\mathrm{QD} 1}=0.71 \mathrm{~ns}, \tau_{\mathrm{QD} 2}=2.31 \mathrm{~ns}, g_{1}^{\max }=29.52 \mathrm{~cm}^{-1}, g_{2}^{\max }=7.92 \mathrm{~cm}^{-1}, \sigma_{2}=10^{-13} \mathrm{~cm}^{2}$, $L=0.114 \mathrm{~cm}$, and $T=300 \mathrm{~K} ; 5 \%$ QD-size fluctuations are assumed. The mirror reflectivity at the ground- and excited-state transition energies is put the same, $R_{1}=R_{2}=0.32$ (as-cleaved facets at both ends), and hence the mirror loss $\beta_{1}=\beta_{2}=10 \mathrm{~cm}^{-1}$. The values of $\tau_{21}$ for different curves (from bottom to top) are $100,10,2$, and $1 \mathrm{ps}$. The corresponding values of $j_{\text {th1 } 1}$ are $21.20,13.60,13.11$, and $13.05 \mathrm{~A} / \mathrm{cm}^{2}$. Because the preceding values of $\tau_{21}$ are much smaller than $\tau_{21}^{\max }=521 \mathrm{ps}$ [Eq. (22)], $P_{1}^{\max }$ is inversely proportional to $\tau_{21}$ [Eq. (25)].

$$
P_{1}(j)=\hbar \omega_{1} \mathrm{v}_{\mathrm{g} 1} \beta_{1} n_{\mathrm{ph} 1}(j) S b=\hbar \omega_{1} \frac{n_{\mathrm{ph} 1}(j)}{\tau_{\mathrm{ph} 1}} S b,
$$

where $\hbar \omega_{1}$ is the energy of photons emitted via ground-state transitions (Fig. 1).

The dependences of $f_{2}$ and $n_{\mathrm{OCL}}$ on $j$ are also easily obtained from those on $n_{\mathrm{ph} 1}$ by converting the variable on the $x$ axis from $n_{\mathrm{ph} 1}$ into $j\left(n_{\mathrm{ph} 1}\right)$.

Fig. 2 shows $n_{\mathrm{ph} 1}$ and $P_{1}$ versus the excess of the injection current density $j$ over the threshold current density $j_{\text {th1 }}$ for ground-state lasing [see Eq. (19) for $j_{\text {th } 1}$ in the following text]. The dependences are plotted for different values of the excited-to-ground-state relaxation time $\tau_{21}$. Experimental and calculated values of $\tau_{21}$ taken from Refs. 18, 20, 21, and 24-31 are presented in Table III.

The LCC was shown to be sublinear in the case of direct capture of carriers from the OCL into single-state QDs. ${ }^{22,23}$ The sublinearity is due to (i) noninstantaneous capture from

TABLE III. Reported values of the intradot relaxation time $\tau_{21}$.

\begin{tabular}{|c|c|c|c|c|}
\hline $\begin{array}{l}\tau_{21} \\
(\mathrm{ps})\end{array}$ & $\begin{array}{l}\text { Temperature } \\
\text { (K) }\end{array}$ & $\begin{array}{l}\text { Energy separation } \\
\text { between the excited } \\
\text { and ground } \\
\text { states in } \\
\text { QDs, } \Delta(\mathrm{meV})\end{array}$ & $\begin{array}{l}\text { Material } \\
\text { system }\end{array}$ & Source \\
\hline 7 & $\begin{array}{l}\text { Room } \\
\text { temperature } \\
\text { (RT) }\end{array}$ & & $\begin{array}{c}\mathrm{InAs} / \mathrm{In}_{0.15} \mathrm{Ga}_{0.85} \mathrm{As} / \\
\mathrm{GaAs}\end{array}$ & 18 and 20 \\
\hline 5.6 & 10 & 60 & $\mathrm{In}(\mathrm{Ga}) \mathrm{As} / \mathrm{GaAs}$ & 21 \\
\hline 1,570 & 12 & 11 & $\mathrm{In}_{0.1} \mathrm{Ga}_{0.9} \mathrm{As} / \mathrm{GaAs} / \mathrm{InP}$ & 24 \\
\hline 19 & & $<2$ & $\mathrm{GaAs} / \mathrm{Al}_{0.3} \mathrm{Ga}_{0.7} \mathrm{As}$ & 25 \\
\hline $2.7-17$ & 12 & 11 & $\mathrm{In}_{0.1} \mathrm{Ga}_{0.9} \mathrm{As} / \mathrm{GaAs} / \mathrm{InP}$ & 26 \\
\hline 150 & RT & 50 & GaAs-AlGaAs & 27 \\
\hline $30-50$ & RT & & $\mathrm{In}_{0.4} \mathrm{Ga}_{0.6} \mathrm{As} / \mathrm{GaAs}$ & 28 \\
\hline $0.6-6$ & 2 & 40 & $\mathrm{InP} / \mathrm{Ga}_{0.5} \mathrm{In}_{0.5} \mathrm{P}$ & 29 \\
\hline 30,100 & & & InGaAs/GaAs & 30 \\
\hline$>30$ & $\begin{array}{l}\text { Liquid } \\
\text { helium }\end{array}$ & & $\mathrm{InAs} / \mathrm{GaAs}$ & 31 \\
\hline
\end{tabular}

the OCL into QDs and (ii) recombination in the OCL, the rate of which is superlinear in carrier density (quadratic or cubic for spontaneous radiative or nonradiative Auger recombination). In the case under study here, as seen from Fig. 2, the output power is more severely impacted-it becomes saturated with increasing $j$. The point is that the capture into the QD lasing state is now a two-step process. In addition to capture delay from the OCL to the QD excitedstate, there is now excited-to-ground-sate relaxation delay. It is the relaxation delay, which now controls the carrier supply to the lasing state and strongly limits the output power by causing its saturation at high injection currents.

\section{Characteristics at the lasing threshold}

Putting $n_{\text {ph1 }}=0$ into Eqs. (7), (10), and (12), the values of $f_{2}$ and $n_{\mathrm{OCL}}$ at the ground-state lasing threshold and the threshold current density are obtained

$$
f_{2, \text { th } 1}=\frac{1}{f_{1}+\left(1-f_{1}\right) \frac{\tau_{12}}{\tau_{21}}}\left(f_{1}+\frac{\tau_{12}}{\tau_{\mathrm{QD} 1}} f_{1}^{2}\right),
$$

$$
\begin{aligned}
n_{\mathrm{OCL}, \mathrm{th} 1}= & n_{2} \frac{f_{2, \mathrm{th} 1}}{1-f_{2, \mathrm{th} 1}}+\frac{1}{\sigma_{2} \mathrm{v}_{\mathrm{n}}} \frac{1}{1-f_{2, \mathrm{th} 1}} \\
& \times\left[\frac{f_{2, \mathrm{th} 1}\left(1-f_{1}\right)}{\tau_{21}}-\frac{f_{1}\left(1-f_{2, \mathrm{th} 1}\right)}{\tau_{12}}+\frac{f_{2, \mathrm{th} 1}^{2}}{\tau_{\mathrm{QD} 2}}\right],
\end{aligned}
$$

$$
\begin{aligned}
j_{\mathrm{th} 1}= & e b B n_{\mathrm{OCL}, \mathrm{th} 1}^{2} \\
& +e N_{\mathrm{S}}\left[\frac{f_{2, \mathrm{th} 1}\left(1-f_{1}\right)}{\tau_{21}}-\frac{f_{1}\left(1-f_{2, \mathrm{th} 1}\right)}{\tau_{12}}+\frac{f_{2, \mathrm{th} 1}^{2}}{\tau_{\mathrm{QD} 2}}\right] .
\end{aligned}
$$

Taking into account Eq. (15), we can write $n_{\mathrm{OCL}, \mathrm{th} 1}$ and $j_{\text {th1 }}$ as

$$
n_{\mathrm{OCL}, \mathrm{th} 1}=n_{2} \frac{f_{2, \mathrm{th} 1}}{1-f_{2, \mathrm{th} 1}}+\frac{1}{\sigma_{2} \mathrm{v}_{\mathrm{n}}} \frac{1}{1-f_{2, \mathrm{th} 1}}\left(\frac{f_{1}^{2}}{\tau_{\mathrm{QD} 1}}+\frac{f_{2, \mathrm{th} 1}^{2}}{\tau_{\mathrm{QD} 2}}\right),
$$




$$
j_{\mathrm{th} 1}=e b B n_{\mathrm{OCL}, \mathrm{th} 1}^{2}+e N_{\mathrm{S}} \frac{f_{1}^{2}}{\tau_{\mathrm{QD} 1}}+e N_{\mathrm{S}} \frac{f_{2, \mathrm{th} 1}^{2}}{\tau_{\mathrm{QD} 2}} .
$$

The first, second, and third terms in the right-hand side of Eq. (19) are the threshold values of the current densities of spontaneous radiative recombination via the OCL states, and ground and excited states in QDs, respectively.

\section{Maximum output power and necessary condition for ground-state lasing}

It is seen from Eq. (7) that, as $f_{2} \rightarrow 1$ (the level occupancy cannot exceed unity) with increasing injection current, the photon density $n_{\mathrm{ph} 1}$ remains finite and tends to its maximum (saturation) value (Fig. 2). Putting $f_{2}=1$ in Eq. (7) gives

$$
n_{\mathrm{ph} 1}^{\max }=\tau_{\mathrm{ph} 1}\left(\frac{1-f_{1}}{\tau_{21}}-\frac{f_{1}^{2}}{\tau_{\mathrm{QD} 1}}\right) \frac{N_{\mathrm{S}}}{b} .
$$

For the maximum (saturation) value of the output power of ground-state lasing, we have

$$
P_{1}^{\max }=\hbar \omega_{1} \frac{n_{\mathrm{ph} 1}^{\max }}{\tau_{\mathrm{ph} 1}} S b=\hbar \omega_{1}\left(\frac{1-f_{1}}{\tau_{21}}-\frac{f_{1}^{2}}{\tau_{\mathrm{QD} 1}}\right) N_{\mathrm{S}} S .
$$

The output power $P_{1}$ approaches its saturation value $P_{1}^{\max }$ according to $(1-\mathrm{const} / \sqrt{j}) P_{1}^{\max }$-see Eq. (A6) in Appendix A for the asymptotic expression for the LCC at high injection currents.

As seen from Eq. (21), the maximum power $P_{1}^{\max }$ of ground-state lasing is a decreasing function of the intradot relaxation time $\tau_{21}$. At a certain value of $\tau_{21}$ given by

$$
\tau_{21}^{\max }=\frac{1-f_{1}}{f_{1}^{2}} \tau_{\mathrm{QD} 1}
$$

$P_{1}^{\max }$ vanishes. Hence the condition for lasing can be formulated as

$$
\tau_{21}<\tau_{21}^{\max } .
$$

As can be seen from Eqs. (15) and (22), if $\tau_{21}=\tau_{21}^{\max }$, the excited-state occupancy is unity already at the groundstate lasing threshold $\left(f_{2, \text { th } 1}=1\right)$; hence $n_{\mathrm{OCL} \text {,th } 1}$ and the threshold current density $j_{\text {th } 1}$ of ground-state lasing [see Eqs. (18) and (19)] become infinitely high.

$\tau_{21}^{\max }$ presents the longest, cut-off value of the intradot relaxation time-no ground-state lasing is possible if $\tau_{21}>\tau_{21}^{\max }$. This cut-off time depends on the ground-state occupancy $f_{1}$. As seen from Eq. (6), $f_{1}$ can range from $1 / 2$ (infinitely long cavity, i.e., no mirror loss, $\beta_{1}=0$ ) to 1 (shortest cavity, $\beta_{1}=g_{1}^{\max }$ ). As seen from (22), $\tau_{21}^{\max }$ decreases from $2 \tau_{\mathrm{QD} 1}$ to 0 as $f_{1}$ varies from $1 / 2$ to 1 . Hence

$$
\tau_{21}^{\text {abs_max }}=2 \tau_{\mathrm{QD} 1}
$$

presents the absolute upper limit for $\tau_{21}$. If $\tau_{21}>\tau_{21}^{\text {abs_max }}$, the lasing is unattainable even in a structure with no mirror loss.

The fact that $\tau_{21}^{\max }=0$ in the device with the shortest cavity means that no lasing is possible in such a device even if intradot relaxation is instantaneous.
With Eq. (22), the maximum value of the stimulated recombination rate per QD, $\frac{b}{N_{\mathrm{S}}} \frac{n_{\mathrm{ph} 1}^{\max }}{\tau_{\mathrm{ph}}}=\frac{1}{S N_{\mathrm{S}}} \frac{P_{1}^{\max }}{\hbar \omega_{1}}$, normalized to the ground-state spontaneous recombination rate per QD, $f_{1}^{2} / \tau_{\mathrm{QD} 1}$, can be written as the following universal decaying function of the normalized relaxation time $\tau_{21} / \tau_{21}^{\max }$ (Fig. 3):

$$
\frac{\frac{b}{N_{\mathrm{S}}} \frac{n_{\mathrm{ph} 1}^{\max }}{\tau_{\mathrm{ph} 1}}}{\frac{f_{1}^{2}}{\tau_{\mathrm{QD} 1}}}=\frac{\frac{1}{S N_{\mathrm{S}}} \frac{P_{1}^{\max }}{\hbar \omega_{1}}}{\frac{f_{1}^{2}}{\tau_{\mathrm{QD} 1}}}=\frac{1}{\frac{\tau_{21}}{\tau_{21}^{\max }}}-1 .
$$

When $\tau_{21} / \tau_{21}^{\max } \ll 1$, the normalized $P_{1}^{\max }$ is inversely proportional to $\tau_{21} / \tau_{21}^{\max }$ (the linear portion of the curve in Fig. 3 in log-scale; see also the horizontal dotted lines in Fig. 2).

Inequality Eq. (23), which is the necessary condition for ground-state lasing, is written in terms of allowed values of $\tau_{21}$ at a given $f_{1}$, i.e., given $g_{1}^{\max }$ and $\beta_{1}$. This condition can be rewritten in terms of allowed values of $g_{1}^{\max }$ at given $\tau_{21}$ and $\beta_{1}$. Thus we obtain

$$
g_{1}^{\max }>\frac{\beta_{1}}{\frac{1}{4}+\sqrt{\frac{1}{16}+\frac{1}{4} \frac{\tau_{21}}{\tau_{\mathrm{QD} 1}}}}-1 .
$$

For the entire range of allowed values of $\tau_{21}$, the right-hand side of Eq. (26) is larger than or equal to $\beta_{1}$. Indeed the denominator of the expression in the right-hand side is less than or equal to unity-it varies from 1 to 0 as $\tau_{21}$ varies from 0 to $\tau_{21}^{\text {abs_max }}=2 \tau_{\mathrm{QD} 1}$.

Hence, when the carrier capture into the QD ground-state is excited-state-mediated, a simple excess of the maximum gain $g_{1}^{\max }$ over the mirror loss $\beta_{1}$ is not sufficient for groundstate lasing to occur. A stronger condition [inequality Eq. (26)] should be satisfied. Only if intradot relaxation is instantaneous, Eq. (26) reduces to the condition $g_{1}^{\max }>\beta_{1}$. The longer is $\tau_{21}$, the higher should be $g_{1}^{\max }$. For Eq. (26) to hold in the case of the slowest intradot relaxation $\left(\tau_{21}=\tau_{21}^{\text {abs_max }}=2 \tau_{\mathrm{QD} 1}\right)$, the maximum gain for ground-state transitions $g_{1}^{\max }$ should be infinitely high or the cavity infinitely long $\left(\beta_{1}=0\right)$.

\section{Internal and external differential quantum efficiencies}

Above the lasing threshold $\left(j \geq j_{\text {th } 1}\right)$, the internal differential quantum efficiency of a semiconductor laser is defined

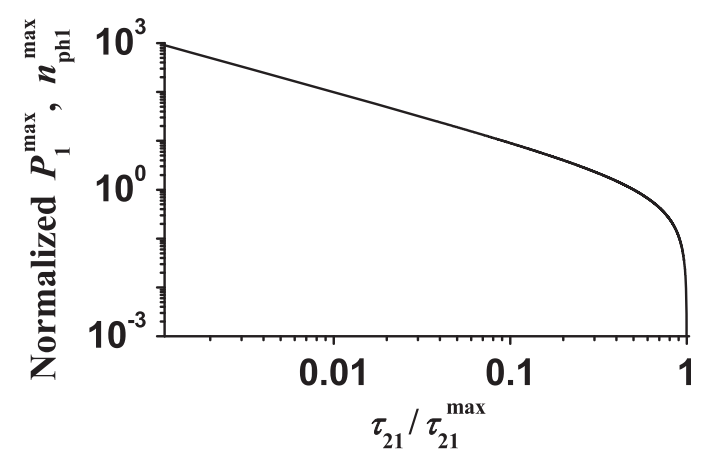

FIG. 3. Normalized maximum output power and photon density [Eq. (25)] of ground-state lasing as a universal function of normalized intradot relaxation time. 
as the fraction of the excess of the injection current over the threshold current that results in stimulated emission

$$
\eta_{\text {int } 1}=\frac{j_{\text {stim } 1}}{j-j_{\text {th1 }}}
$$

where

$$
j_{\mathrm{stim} 1}=e b \frac{n_{\mathrm{ph} 1}}{\tau_{\mathrm{ph} 1}}
$$

is the current density of stimulated recombination via the ground-state in QDs.

With Eqs. (13) and (19) for $j$ and $j_{\text {th1 }}$, Eq. (27) becomes

$$
\eta_{\mathrm{int} 1}=\frac{1}{1+\frac{\tau_{12}}{\tau_{\mathrm{QD} 2}} \frac{f_{2}+f_{2, \mathrm{th} 1}}{f_{1}+\left(1-f_{1}\right) \frac{\tau_{12}}{\tau_{21}}}+\frac{B\left(n_{\mathrm{OCL}}^{2}-n_{\mathrm{OCL}, \mathrm{th} 1}^{2}\right)}{\frac{n_{\mathrm{ph} 1}}{\tau_{\mathrm{ph} 1}}}}
$$

In Eq. (29), Eqs. (7) and (15) for $f_{2}$ and $f_{2, \text { th } 1}$ were used.

The external differential quantum efficiency is defined as

$$
\eta_{\mathrm{ext} 1}=\frac{1}{\frac{\hbar \omega_{1}}{e}} \frac{\partial P_{1}}{\partial I}=e b \frac{1}{\tau_{\mathrm{ph} 1}} \frac{\partial n_{\mathrm{ph} 1}}{\partial j}=\frac{\partial j_{\mathrm{stim} 1}}{\partial j},
$$

where $I=S j$ is the injection current. With Eqs. (7), (10), and (11), we have for $\eta_{\text {ext1 }}$

$$
\begin{aligned}
\eta_{\mathrm{ext} 1}= & \left\{1+2 \frac{\tau_{12}}{\tau_{\mathrm{QD} 2}} \frac{f_{2}}{f_{1}+\left(1-f_{1}\right) \frac{\tau_{12}}{\tau_{21}}}+2 \tau_{12} \frac{b}{N_{\mathrm{S}}} B n_{\mathrm{OCL}}\right. \\
& \times \frac{1}{1-f_{2}} \frac{1}{f_{1}+\left(1-f_{1}\right) \frac{\tau_{12}}{\tau_{21}}} \\
& \left.\times\left[n_{\mathrm{OCL}}+n_{2}+\frac{1}{\sigma_{2} \mathrm{v}_{\mathrm{n}}}\left(\frac{1-f_{1}}{\tau_{21}}+\frac{f_{1}}{\tau_{12}}+\frac{2 f_{2}}{\tau_{\mathrm{QD} 2}}\right)\right]\right\}^{-1} .
\end{aligned}
$$

In Eqs. (29) and (31), $f_{2}, n_{\mathrm{OCL}}$, and $n_{\mathrm{ph} 1}$ are functions of the injection current density $j$.

Even at the lasing threshold, the internal and external efficiencies (being equal to each other) are less than unity (Fig. 4).

At $j>j_{\text {th } 1}, \eta_{\text {ext1 }}$ is smaller than $\eta_{\text {int1 }}$ (Fig. 4). Both efficiencies decrease rapidly with $j$ (Fig. 4). The asymptotic expressions for them at high $j$ are derived in Appendix $\mathrm{A}-$ $\eta_{\text {int1 }}$ and $\eta_{\text {ext1 }}$ decay as $1 / j$ and $1 / j^{3 / 2}$, respectively [Eqs. (A8) and (A9)].

The shorter the intradot relaxation time $\tau_{21}$, the higher $\eta_{\text {int1 }}$ and $\eta_{\text {ext1 }}$ (Fig. 4). The limiting case of instantaneous relaxation $\left(\tau_{21}=0\right)$ is considered in Appendix B.

We considered in Section II the situation when the lasing occurs via ground-state transitions only. This means that with increasing pump current, the lasing condition will never be satisfied for excited-state transitions. A sufficient condition for this is the inequality

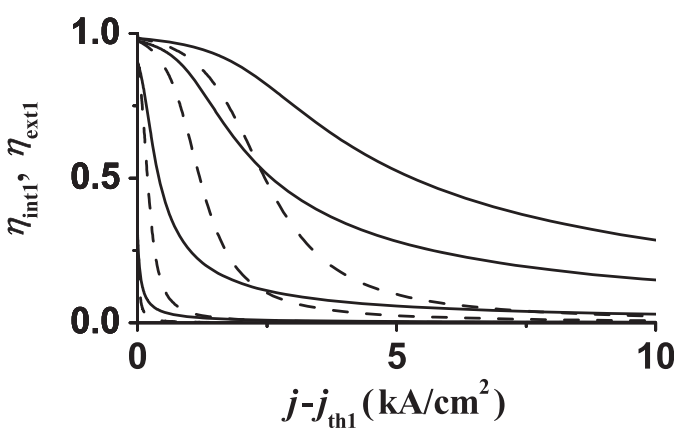

FIG. 4. Internal and external differential quantum efficiencies (solid and dashed curves, respectively) against excess injection current density. The values of $\tau_{21}$ for different curves (from bottom to top) are 100, 10, 2, and 1 ps.

$$
g_{2}^{\max }<\beta_{2}
$$

where $g_{2}^{\max }$ and $\beta_{2}$ are the maximum modal gain and mirror loss for excited-state transitions. For calculations in this section, we used 29.52 and $7.92 \mathrm{~cm}^{-1}$ for $g_{1}^{\max }$ and $g_{2}^{\max }$ and the same value $\left(10 \mathrm{~cm}^{-1}\right)$ for $\beta_{1}$ and $\beta_{2}$. Other parameters are presented in the caption to Fig. 2.

\section{GROUND- AND EXCITED-STATE LASING: HIGH GAIN FOR BOTH GROUND- AND EXCITED-STATE TRANSITIONS}

The condition Eq. (32) of low gain for excited-state transitions is not always satisfied. When the inequality reverse to Eq. (32) holds

$$
g_{2}^{\max }>\beta_{2}
$$

excited-state lasing occurs above a certain injection current (threshold current for excited-state lasing). Depending on the maximum gain $g_{1}^{\max }$ for ground-state transitions, two situations are possible. If $g_{1}^{\max }$ is high enough [the criterion is formulated in the following text-see Eq. (40)], ground-state lasing turns on first with increasing pump current and then so does excited-state lasing. Such a situation is considered in this section; for calculations here, we use $g_{1}^{\max }=29.52 \mathrm{~cm}^{-1}$ and $g_{2}^{\max }=12 \mathrm{~cm}^{-1}$; other parameters are presented in the caption to Fig. 5. If $g_{1}^{\max }$ is low, lasing will occur via excited-state transitions only. Such a situation is considered in Section IV.

\section{A. Above ground-state lasing threshold and below excited-state lasing threshold: Ground-state lasing only}

All the equations and analysis of Section II apply in this case of $j_{\text {th } 1}<j<j_{\text {th } 2}$, where $j_{\text {th } 1}$ is the threshold current density for ground-state lasing given by Eq. (19), and $j_{\text {th2 }}$ is the threshold current density for excited-state lasing given by Eq. (46) in the following text. Particularly, with increasing pump current density above $j_{\text {th } 1}$, the photon density and output power of ground-state lasing increase from zero, and the excited-state occupancy increases from its value $f_{2 \text {,th } 1}$ [given by Eq. (15)]; $f_{2}$ and $n_{\mathrm{ph} 1}$ are related by Eq. (7). The increase in $n_{\mathrm{ph} 1}$ and $f_{2}$ continues up to the onset of excited-state lasing (Fig. 5). 


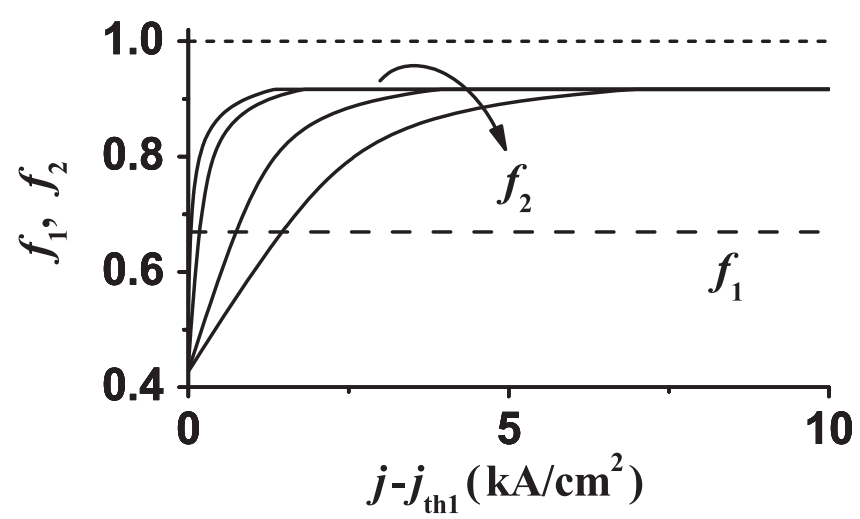

FIG. 5. Ground- (horizontal dashed line) and excited-state (solid curves) occupancies in QDs against excess injection current density. The horizontal short-dashed line indicates the maximum possible value (unity) for the level occupancies. In Figs. 5-12, the parameters are as follows (those not listed here are the same as in the caption to Fig. 2): $\tau_{\mathrm{QD} 2}=1.53 \mathrm{~ns}$ and $g_{2}^{\max }=12 \mathrm{~cm}^{-1}$. The values of $\tau_{21}$ for different curves (from left to right) are $100,10,2$, and $1 \mathrm{ps}$. The corresponding values of $j_{\text {th1 }}$ are 22.00, 13.60, 13.11 , and $13.05 \mathrm{~A} / \mathrm{cm}^{2}$, and the values of $j_{\mathrm{th} 2}$ are $1.34 \times 10^{3}, 1.60 \times 10^{3}$, $2.71 \times 10^{3}$, and $4.10 \times 10^{3} \mathrm{~A} / \mathrm{cm}^{2}$. The pinning of $f_{2}$ occurs at $j=j_{\text {th } 2}$.

\section{B. Above excited-state lasing threshold: Simultaneous ground- and excited-state lasing}

The set of the rate Eqs. (1)-(4) should now be added by the rate equation for photons emitted via excited-state stimulated transitions. This equation is similar to Eq. (4)

$$
\mathrm{v}_{\mathrm{g} 2} g_{2}^{\max }\left(2 f_{2}-1\right) n_{\mathrm{ph} 2}-\mathrm{v}_{\mathrm{g} 2} \beta_{2} n_{\mathrm{ph} 2}=0
$$

where $\mathrm{v}_{\mathrm{g} 2}$ and $n_{\mathrm{ph} 2}$ are the group velocity and density of photons emitted via excited-state stimulated transitions.

At and above $j_{\text {th2 }}$ (i.e., when $n_{\mathrm{ph} 2} \neq 0$ ), Eq. (34) reads as the condition of equality of the gain for excited-state transitions to the mirror loss (condition for excited-state lasing), from which we have

$$
f_{2}=f_{2, \mathrm{th} 2}=\frac{1}{2}\left(1+\frac{\beta_{2}}{g_{2}^{\max }}\right) .
$$

Similarly to the ground-state occupancy above $j_{\text {th } 1}$, the excited-state occupancy above $j_{\text {th2 }}$ is also pinned (Fig. 5).

\section{Pinning of output power and necessary condition for ground-state lasing}

Because $n_{\mathrm{ph} 1}$ is related to $f_{2}$ [see Eq. (7), which is just another way of writing the rate Eq. (3)], it follows immediately from pinning of $f_{2}$ that $n_{\mathrm{ph} 1}$ (and hence $P_{1}$-see Fig. 6) is also pinned above the excited-state lasing threshold. The pinning value of $n_{\mathrm{ph} 1}$ is found from Eq. (7) by putting $f_{2}=f_{2, \text { th2 }}$ there

$$
n_{\mathrm{ph} 1}^{\mathrm{pin}}=\tau_{\mathrm{ph} 1}\left\{\frac{f_{2, \mathrm{th} 2}\left[f_{1}+\left(1-f_{1}\right) \frac{\tau_{12}}{\tau_{21}}\right]-f_{1}}{\tau_{12}}-\frac{f_{1}^{2}}{\tau_{\mathrm{QD} 1}}\right\} \frac{N_{\mathrm{S}}}{b} .
$$

For the pinning value of $P_{1}$, we thus have

$$
\begin{aligned}
P_{1}^{\mathrm{pin}} & =\hbar \omega_{1} \frac{n_{\mathrm{ph} 1}^{\mathrm{pin}}}{\tau_{\mathrm{ph} 1}} S b \\
& =\hbar \omega_{1}\left\{\frac{f_{2, \mathrm{th} 2}\left[f_{1}+\left(1-f_{1}\right) \frac{\tau_{12}}{\tau_{21}}\right]-f_{1}}{\tau_{12}}-\frac{f_{1}^{2}}{\tau_{\mathrm{QD} 1}}\right\} N_{\mathrm{S}} S .
\end{aligned}
$$

Compare Eqs. (36) and (37) with Eqs. (20) and (21) for $n_{\mathrm{ph} 1}^{\max }$ and $P_{1}^{\max }$. Because $f_{2, \text { th } 2}<1$, the pinning value of the output power of ground-state lasing $P_{1}^{\text {pin }}$ is lower than the saturation value $P_{1}^{\max }$. If $f_{2, \text { th } 2} \rightarrow 1, P_{1}^{\text {pin }} \rightarrow P_{1}^{\max }$.

For ground-state lasing to occur, $P_{1}^{\text {pin }}$ must be positive. At a certain value of $\tau_{21}$ given by

$$
\tau_{21}^{\max ^{\prime}}=\frac{f_{2, \mathrm{th} 2}-f_{1}\left[f_{2, \mathrm{th} 2}+\left(1-f_{2, \mathrm{th} 2}\right) \frac{\tau_{21}}{\tau_{12}}\right]}{f_{1}^{2}} \tau_{\mathrm{QD} 1},
$$

$P_{1}^{\text {pin }}$ vanishes. Hence the condition for ground-state lasing can now be formulated as

$$
\tau_{21}<\tau_{21}^{\max ^{\prime}}
$$

$\tau_{21}^{\max ^{\prime}}$ is the cut-off value of $\tau_{21}$ in the case when excited-state lasing is also possible-there can be no ground-state lasing if $\tau_{21}>\tau_{21}^{\max ^{\prime}}$ [compare Eq. (38) with Eq. (22) for $\tau_{21}^{\max }$ ]. Because $f_{2 \text {,th } 2}<1$, this cut-off value (shown by the vertical dotted line in Fig. 7) is shorter than $\tau_{21}^{\max }$. If $f_{2, \text { th } 2} \rightarrow 1$, $\tau_{21}^{\max ^{\prime}} \rightarrow \tau_{21}^{\max }$

Inequality Eq. (39) presents the necessary condition for ground-state lasing in terms of allowed values of $\tau_{21}$ at given $f_{1}$ and $f_{2, \text { th2 }}$, i.e., given $\beta_{1}, g_{1}^{\max }, \beta_{2}$, and $g_{2}^{\max }$. It can be rewritten in terms of allowed values of $g_{1}^{\max }$ at given $\tau_{21}$ as follows:

$$
g_{1}^{\max }>\frac{\beta_{1}}{\frac{1}{4}\left[f_{2, \text { th } 2}+\left(1-f_{2, \text { th } 2}\right) \frac{\tau_{21}}{\tau_{12}}\right]+\sqrt{\frac{1}{16}\left[f_{2, \text { th } 2}+\left(1-f_{2, \text { th } 2}\right) \frac{\tau_{21}}{\tau_{12}}\right]^{2}+\frac{1}{4} \frac{\tau_{21}}{\tau_{\mathrm{QD} 1}} f_{2, \mathrm{th} 2}}}-1
$$




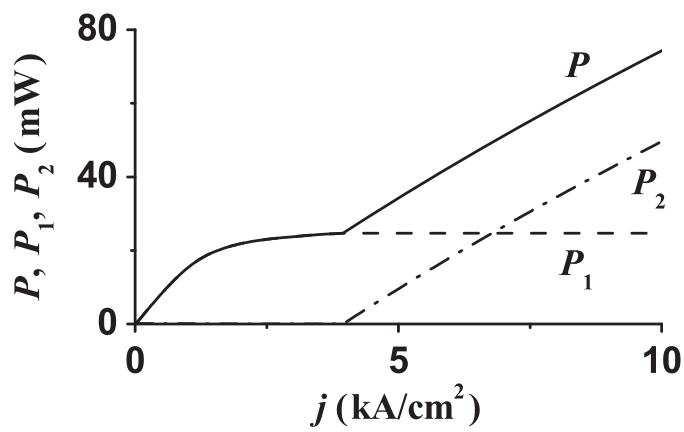

FIG. 6. Total power (solid curve) and powers of ground- (dashed curve) and excited-state (dash-dotted curve) lasing against injection current density. The kink in the curve for the total power occurs at $j=j_{\text {th2 }}$. $\tau_{21}=2 \mathrm{ps}$.

where $f_{1}$ and $f_{2, \text { th2 }}$ are given by Eqs. (6) and (35), correspondingly. If $f_{2, \text { th } 2} \rightarrow 1$, inequality Eq. (40) reduces to Eq. (26).

As in the case of only ground-state lasing (Section II), the longer is $\tau_{21}$, the higher should be $g_{1}^{\max }$. At $\tau_{21}=0$, Eq. (40) reduces to a simple condition $g_{1}^{\max }>\beta_{1}$. At $\tau_{21}=\tau_{21}^{\max ^{\prime}}$, the denominator in the right-hand side of Eq. (40) becomes equal to 0 , and hence $g_{1}^{\max }$ should be infinitely high to have ground-state lasing.

In terms of the normalized relaxation time $\tau_{21} / \tau_{21}^{\max ^{\prime}}$, the pinning value of the ground-state stimulated recombination rate per QD, $\frac{b}{N_{\mathrm{S}}} \frac{p_{\mathrm{ph} 1}^{\mathrm{ph} 1}}{\tau_{\mathrm{ph}}}=\frac{1}{S N_{\mathrm{S}}} \frac{P_{\mathrm{s}}^{\mathrm{pin}}}{\hbar \omega_{1}}$, normalized to the ground-state spontaneous recombination rate per $\mathrm{QD}, f_{1}^{2} / \tau_{\mathrm{QD} 1}$, is given by the same function as the normalized maximum value of the ground-state stimulated recombination rate per QD in terms of $\tau_{21} / \tau_{21}^{\max }$ [Section II, Eq. (25) and Fig. 3].

\section{Free-carrier density in the OCL, photon density, and output power of excited-state lasing}

For $j \geq j_{\text {th2 } 2}$, instead of Eq. (2), the following modified rate equation should be used, which includes the rate of stimulated radiative recombination transitions via the QD excitedstate (the last term in the left-hand side in the following text):

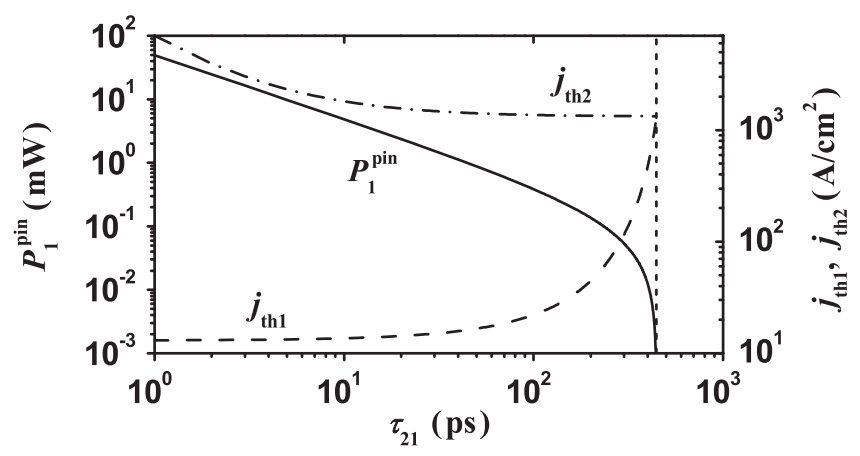

FIG. 7. Output power of ground-state lasing at which excited-state lasing starts (solid curve, left axis) and threshold current densities for ground- and excited-state lasing (dashed and dash-dotted curves, right axis) against intradot relaxation time. The vertical short-dashed line marks $\tau_{21}^{\max }=445 \mathrm{ps}$ at which the ground-state lasing becomes unattainable.

$$
\begin{gathered}
\sigma_{2} \mathrm{v}_{\mathrm{n}} \frac{N_{\mathrm{S}}}{b}\left(1-f_{2, \mathrm{th} 2}\right) n_{\mathrm{OCL}}-\sigma_{2} \mathrm{v}_{\mathrm{n}} n_{2} \frac{N_{\mathrm{S}}}{b} f_{2, \mathrm{th} 2} \\
+\frac{N_{\mathrm{S}}}{b} \frac{f_{1}\left(1-f_{2, \mathrm{th} 2}\right)}{\tau_{12}}-\frac{N_{\mathrm{S}}}{b} \frac{f_{2, \mathrm{th} 2}\left(1-f_{1}\right)}{\tau_{21}} \\
-\frac{N_{\mathrm{S}}}{b} \frac{f_{2, \mathrm{th} 2}^{2}}{\tau_{\mathrm{QD} 2}}-\mathrm{v}_{\mathrm{g} 2} g_{2}^{\max }\left(2 f_{2, \mathrm{th} 2}-1\right) n_{\mathrm{ph} 2}=0 .
\end{gathered}
$$

Using Eqs. (35) and (36), we have from Eq. (41) for the free carrier density in the $\mathrm{OCL}$

$$
\begin{aligned}
n_{\mathrm{OCL}}= & n_{2} \frac{f_{2, \mathrm{th} 2}}{1-f_{2, \mathrm{th} 2}}+\frac{1}{\sigma_{2} \mathrm{v}_{\mathrm{n}}} \frac{1}{1-f_{2, \mathrm{th} 2}} \\
& \times\left(\frac{f_{1}^{2}}{\tau_{\mathrm{QD} 1}}+\frac{f_{2, \mathrm{th} 2}^{2}}{\tau_{\mathrm{QD} 2}}+\frac{b}{N_{\mathrm{S}}} \frac{n_{\mathrm{ph} 1}^{\mathrm{pin}}}{\tau_{\mathrm{ph} 1}}+\frac{b}{N_{\mathrm{S}}} \frac{n_{\mathrm{ph} 2}}{\tau_{\mathrm{ph} 2}}\right),
\end{aligned}
$$

where we introduced the lifetime in the cavity for photons emitted via excited-state transitions

$$
\tau_{\mathrm{ph} 2}=\frac{1}{\mathrm{v}_{\mathrm{g} 2} \beta_{2}}=\frac{1}{\mathrm{v}_{\mathrm{g} 2} g_{2}^{\max }\left(2 f_{2, \mathrm{th} 2}-1\right)} .
$$

Using Eq. (41), we have from Eq. (1) for the injection current density

$$
j=e b B n_{\mathrm{OCL}}^{2}+e N_{\mathrm{S}} \frac{f_{1}^{2}}{\tau_{\mathrm{QD} 1}}+e N_{\mathrm{S}} \frac{f_{2, \mathrm{th} 2}^{2}}{\tau_{\mathrm{QD} 2}}+e b \frac{n_{\mathrm{ph} 1}^{\mathrm{pin}}}{\tau_{\mathrm{ph} 1}}+e b \frac{n_{\mathrm{ph} 2}}{\tau_{\mathrm{ph} 2}},
$$

where $n_{\mathrm{OCL}}$ is given by Eq. (42).

At the excited-state lasing threshold $\left(n_{\mathrm{ph} 2}=0\right)$, we have from Eqs. (42) and (44)

$$
\begin{aligned}
& n_{\mathrm{OCL}, \mathrm{th} 2}=n_{2} \frac{f_{2, \mathrm{th} 2}}{1-f_{2, \mathrm{th} 2}} \\
& +\frac{1}{\sigma_{2} \mathrm{v}_{\mathrm{n}}} \frac{1}{1-f_{2, \mathrm{th} 2}}\left(\frac{f_{1}^{2}}{\tau_{\mathrm{QD} 1}}+\frac{f_{2, \mathrm{th} 2}^{2}}{\tau_{\mathrm{QD} 2}}+\frac{b}{N_{\mathrm{S}}} \frac{n_{\mathrm{ph} 1}^{\mathrm{pin}}}{\tau_{\mathrm{ph} 1}}\right), \\
& j_{\mathrm{th} 2}=e b B n_{\mathrm{OCL}, \mathrm{th} 2}^{2}+e N_{\mathrm{S}} \frac{f_{1}^{2}}{\tau_{\mathrm{QD} 1}}+e N_{\mathrm{S}} \frac{f_{2, \mathrm{th} 2}^{2}}{\tau_{\mathrm{QD} 2}}+e b \frac{n_{\mathrm{ph} 1}^{\mathrm{p} \text { in }}}{\tau_{\mathrm{ph} 1}} \text {. }
\end{aligned}
$$

Compare Eq. (46) for $j_{\text {th2 }}$ with Eq. (19) for $j_{\text {th } 1}$. Because excited-state lasing follows ground-state lasing, this explains why the current density of stimulated recombination via the ground-state in QDs enters as a component [the last term in the right-hand side of Eq. (46)] into the expression for the threshold current density $j_{\text {th } 2}$ for excited-state lasing.

As seen from Eq. (35), $f_{2 \text {,th } 2}$ is unaffected by $\tau_{21}$ (Fig. 5). Hence, as it follows from Eq. (36), $n_{\mathrm{ph} 1}^{\mathrm{pin}}$ decreases with increasing $\tau_{21}$; consequently, $n_{\mathrm{OCL} \text {,th } 2}$ and $j_{\text {th2 } 2}$ decrease [see Eqs. (45) and (46)]. Conversely, $n_{\mathrm{OCL}, \mathrm{th} 1}$ and $j_{\text {th } 1}$ increase with $\tau_{21}$. The opposite tendencies in $j_{\text {th1 } 1}$ and $j_{\text {th2 } 2}$ are easily understood-increasing $\tau_{21}$, i.e., delaying the excited-to-groundstate relaxation, hinders the ground-state lasing (hence $j_{\text {th1 }}$ increases) while making for excited-state lasing (that is why $j_{\text {th2 }}$ decreases). Hence as $\tau_{21}$ increases, $j_{\text {th1 }}$ and $j_{\text {th2 } 2}$ approach each other (Fig. 7). At $\tau_{21}=\tau_{21}^{\max }$ [see Eq. (38) for $\left.\tau_{21}^{\max }\right], n_{\mathrm{ph} 1}^{\text {pin }}$ becomes zero and $f_{2, \text { th2 } 2}, n_{\mathrm{OCL} \text {,th2, }}$ and $j_{\text {th2 }}$ become equal to 
$f_{2, \text { th } 1}, n_{\mathrm{OCL}, \mathrm{th} 1}$, and $j_{\text {th1 } 1}$, respectively (Fig. 7). In contrast to the case of ground-state lasing only (wherein $j_{\text {th } 1} \rightarrow \infty$ as $\tau_{21} \rightarrow \tau_{21}^{\max }$ ), here $j_{\text {th1 }}$ remains finite at $\tau_{21}=\tau_{21}^{\max ^{\prime}}$ (Fig. 7). As $\tau_{21} \rightarrow 0, n_{\mathrm{ph} 1}^{\mathrm{pin}} \rightarrow \infty$ [Eq. (36)], $n_{\mathrm{OCL}, \mathrm{th} 2} \rightarrow \infty$ [Eq. (45)], and $j_{\text {th2 }} \rightarrow \infty$ [Eq. (46) and Fig. 7], i.e., the excited-state lasing becomes unattainable - there can be ground-state lasing only with $n_{\mathrm{OCL} \text {,th1 }}$ and $j_{\text {th1 }}$ being given by Eqs. (B2) and (B3) (wherein $n_{\mathrm{ph} 1}$ is put zero).

The current density of stimulated recombination via the excited-state in QDs

$$
j_{\mathrm{stim} 2}=e b \frac{n_{\mathrm{ph} 2}}{\tau_{\mathrm{ph} 2}}
$$

is calculated in Appendix C. With Eq. (C5) for $j_{\text {stim2 }}$, the output power of excited-state lasing as a function of the injection current density is

$$
\begin{aligned}
P_{2}= & \hbar \omega_{2} \mathrm{v}_{\mathrm{g} 2} \beta_{2} n_{\mathrm{ph} 2} S b=\frac{\hbar \omega_{2}}{e} j_{\mathrm{stim} 2} S \\
= & \frac{\hbar \omega_{2}}{e} \frac{1}{\frac{1}{2}+\frac{j_{\mathrm{th} 2}^{\mathrm{OCL}}}{j_{\mathrm{capt}, \mathrm{th} 2}}+\sqrt{\left(\frac{1}{2}+\frac{j_{\mathrm{th} 2}^{\mathrm{OCL}}}{j_{\mathrm{capt}, \mathrm{th} 2}}\right)^{2}+\frac{j_{\mathrm{th} 2}^{\mathrm{OCL}}}{j_{\mathrm{capt}, \mathrm{th} 2}} \frac{j-j_{\mathrm{th} 2}}{j_{\mathrm{capt}, \mathrm{th} 2}}}} \\
& \times\left(j-j_{\mathrm{th} 2}\right) S,
\end{aligned}
$$

where

$$
j_{\text {capt }, \text { th } 2}=e \sigma_{2} \mathrm{v}_{\mathrm{n}} n_{\mathrm{OCL}, \mathrm{th} 2}\left(1-f_{2, \mathrm{th} 2}\right) N_{\mathrm{S}}
$$

is the current density of carrier capture from the OCL into the excited-state in QDs at $j=j_{\text {th2 }}$, and

$$
j_{\mathrm{th} 2}^{\mathrm{OCL}}=e b B n_{\mathrm{OCL}, \mathrm{th} 2}^{2}
$$

is the spontaneous radiative recombination current density in the OCL at $j=j_{\text {th2 }}$.

Because the energies of photons emitted via the groundand excited-state transitions are not the same, the total optical power $P=P_{1}^{\text {pin }}+P_{2}$ is not directly proportional to the total photon density $n_{\mathrm{ph}}=n_{\mathrm{ph} 1}^{\mathrm{pin}}+n_{\mathrm{ph} 2}$ at $j>j_{\mathrm{th} 2}$.

Eq. (44) can be rewritten as

$$
j_{\mathrm{stim} 1}^{\mathrm{pin}}+j_{\mathrm{stim} 2}=j-\left(e b B n_{\mathrm{OCL}}^{2}+e N_{\mathrm{S}} \frac{f_{1}^{2}}{\tau_{\mathrm{QD} 1}}+e N_{\mathrm{S}} \frac{f_{2, \mathrm{th} 2}^{2}}{\tau_{\mathrm{QD} 2}}\right) \text {, }
$$

where

$$
j_{\mathrm{stim} 1}^{\mathrm{pin}}=e b \mathrm{v}_{g 1} \beta_{1} n_{\mathrm{ph} 1}^{\mathrm{pin}}=e b \frac{n_{\mathrm{ph} 1}^{\mathrm{pin}}}{\tau_{\mathrm{ph} 1}}
$$

is the current density of stimulated recombination via the ground-state in QDs above the excited-state lasing threshold with $n_{\mathrm{ph} 1}^{\text {pin }}$ being given by Eq. (36). Because $f_{2, \text { th2 }}$ is unaffected by $\tau_{21}$ [see Eq. (35)], as it immediately follows from the rate Eq. (1), so is $n_{\mathrm{OCL}}$; this is also seen from Fig. 8-the curves for $n_{\mathrm{OCL}}$ calculated at different values of $\tau_{21}$ merge together above $j_{\text {th2 }}$. Hence none of the quantities $n_{\mathrm{OCL}}, f_{1}$, and $f_{2, \text { th } 2}$ in

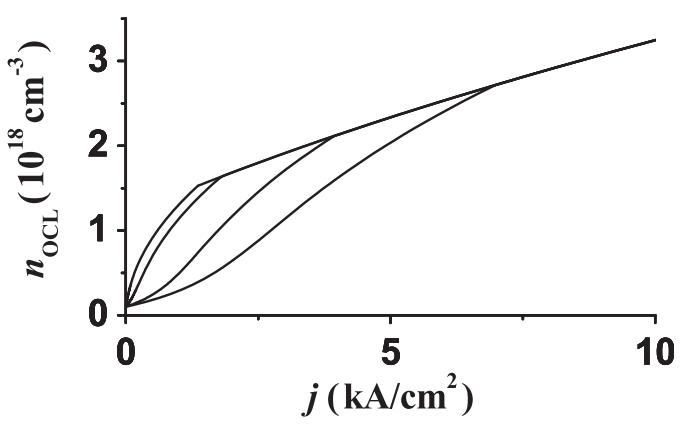

FIG. 8. Free carrier density in the OCL against injection current density. The values of $\tau_{21}$ for different curves (from left to right) are 100,10,2, and 1 ps. The kinks in the curves occur at $j=j_{\text {th2 }}$.

the right-hand side of Eq. (51) depends on $\tau_{21}$. Therefore the spontaneous recombination current densities [via the OCL states and ground and excited states in QDs- the three terms in the brackets in the right-hand-side of Eq. (51)] do not depend on $\tau_{21}$ at $j \geq j_{\text {th2 }}$. Thus the total stimulated recombination current density is also independent of $\tau_{21}$ though both $j_{\text {stim } 1}^{\text {pin }}$ and $j_{\text {stim } 2}$ depend on $\tau_{21}$ on their own.

Hence, as seen from Eq. (51), the sum of the photon densities of ground- and excited-state lasing, each weighted by its own reciprocal lifetime in the cavity, does not depend on $\tau_{21}$ at $j \geq j_{\text {th2 }}$. The following expression is obtained for this sum:

$$
\begin{aligned}
\frac{n_{\mathrm{ph} 1}^{\mathrm{pin}}}{\tau_{\mathrm{ph} 1}}+\frac{n_{\mathrm{ph} 2}}{\tau_{\mathrm{ph} 2}}= & \frac{1}{e b}\left(j_{\mathrm{stim} 1}^{\mathrm{pin}}+j_{\mathrm{stim} 2}\right) \\
= & \frac{1}{e b}\left\{\frac{j_{\mathrm{capt}, \mathrm{th} 2}^{2}}{j_{\mathrm{th} 2}^{\mathrm{OCL}}}\left[\sqrt{\frac{1}{4}+\frac{j_{\mathrm{th} 2}^{\mathrm{OCL}}}{j_{\mathrm{capt}, \mathrm{th} 2}^{2}}\left(j_{\mathrm{esc} 2}^{\mathrm{OCL}}+j\right)}-\frac{1}{2}\right]\right. \\
& \left.-j_{\mathrm{esc} 2}^{\mathrm{OCL}}-e N_{\mathrm{S}} \frac{f_{1}^{2}}{\tau_{\mathrm{QD} 1}}-e N_{\mathrm{S}} \frac{f_{2, \mathrm{th} 2}^{2}}{\tau_{\mathrm{QD} 2}}\right\}=\operatorname{const}\left(\tau_{21}\right),
\end{aligned}
$$

where

$$
j_{\mathrm{esc} 2}^{\mathrm{OCL}}=e \sigma_{2} \mathrm{v}_{\mathrm{n}} n_{2} f_{2, \mathrm{th} 2} N_{\mathrm{S}}
$$

is the current density of carrier escape from the excited-state in QDs to the OCL.

If $\tau_{\mathrm{ph} 1}=\tau_{\mathrm{ph} 2}$, the total photon density $n_{\mathrm{ph} 1}^{\mathrm{pin}}+n_{\mathrm{ph} 2}$ is independent of $\tau_{21}$ [Fig. 9(a)]. Because, as mentioned in the preceding text, the total power $P$ is not directly proportional to the total photon density, there is a slight dependence of $P$ on $\tau_{21}$ even in this case [Fig. 9(b)].

The kinks in the curves in Fig. 9 occur at $j=j_{\text {th } 2}$. As seen from the figure, with increasing $\tau_{21}$, the contribution of ground-state lasing to the total output power decreases. For $\tau_{21}=100 \mathrm{ps}$, this contribution is negligible. This is also seen from Fig. 10 showing the ratio of $n_{\mathrm{ph} 2}$ to $n_{\mathrm{ph} 1}$ at $j \geq j_{\mathrm{th} 2}$.

For $\tau_{21}=2$ ps, Fig. 6 shows the total power $P$ and the powers of ground- and excited-state lasing $P_{1}$ and $P_{2}$, respectively.

We should emphasize here that other factors, which are not included into our theoretical model, may also affect the LCC of a laser in the presence of excited states in QDs. Thus, experimental LCCs both with (see, e.g., Refs. 1, 8, 12, 16, 17, 19, and 20) 

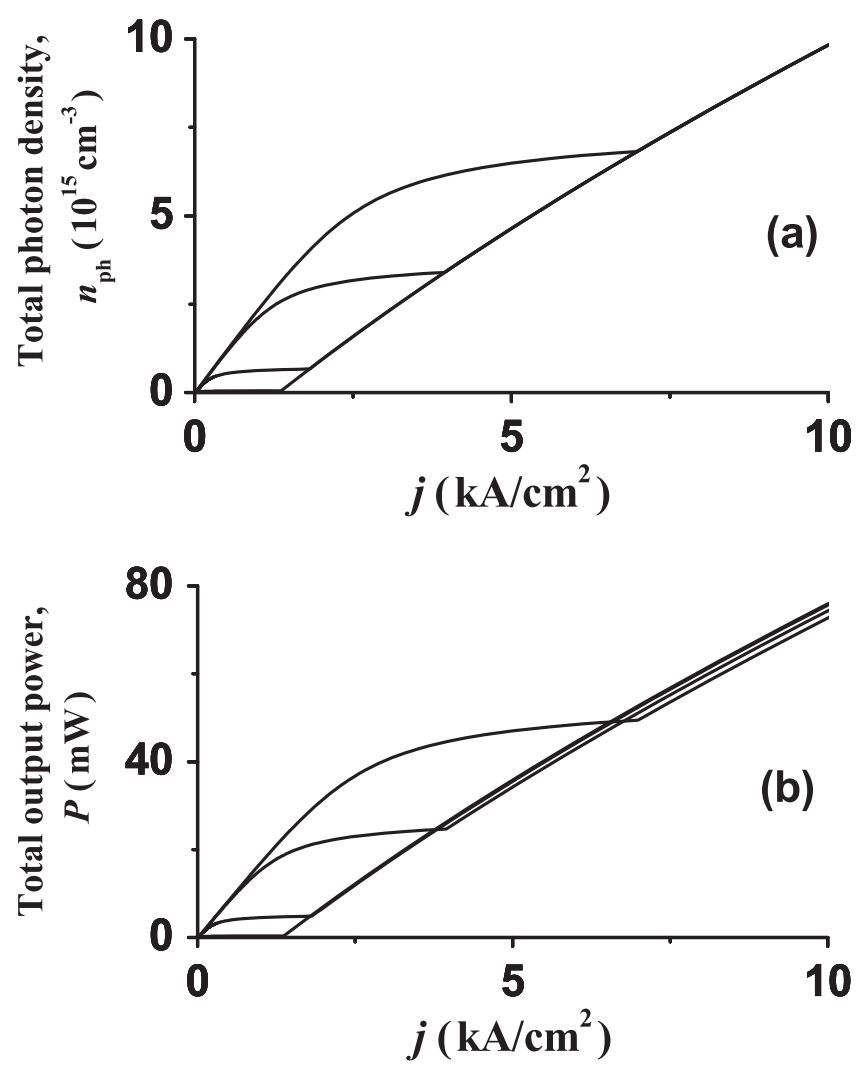

FIG. 9. Total photon density (a) and output power (b) of both ground- and excited-state lasing against injection current density. The kinks in the curves occur at $j=j_{\text {th2 } 2}$ and show $n_{\mathrm{ph} 1}^{\mathrm{pin}}$ and $P_{1}^{\mathrm{pin}}$ [see Eqs. (36) and (37)]. The values of $\tau_{21}$ for different curves (from bottom to top) are 100, 10, 2, and $1 \mathrm{ps}$.

and without ${ }^{4,5,7}$ kinks were reported. Furthermore, even in the presence of a kink, the dependence of the ground-state power on the pump current is not always the same. It remains constant above the excited-state lasing threshold in some structures; ${ }^{8,19}$ in others, the ground-state power approaches its maximum and then rollover occurs. $1,4,7,12,16,17$

\section{Internal and external differential quantum efficiencies}

For $j_{\text {th1 }} \leq j<j_{\text {th2 }}$, the internal quantum efficiency can be introduced for ground-state lasing only [see Eq. (29)]. Above $j_{\text {th2 }}$, the internal efficiency can be introduced for both ground- and excited-state lasing.

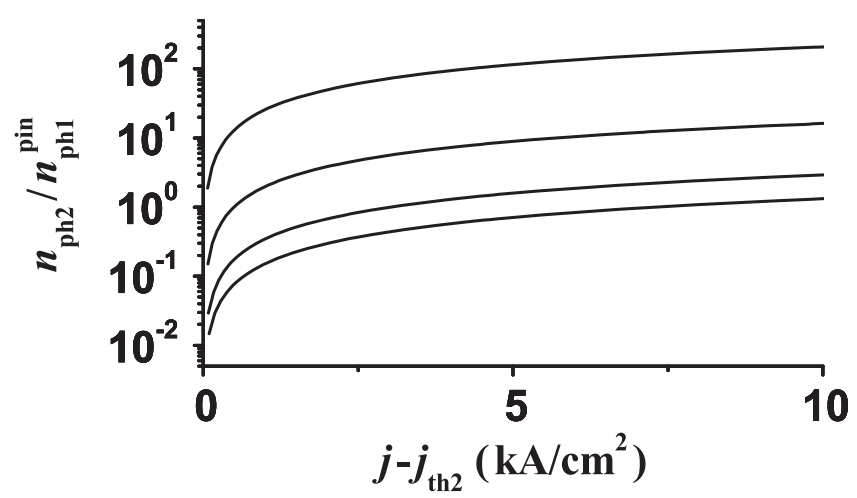

FIG. $10 . n_{\mathrm{ph} 2} / n_{\mathrm{ph} 1}^{\mathrm{pin}}$ ratio against excess of the injection current density over the threshold current density for excited-state lasing. The values of $\tau_{21}$ for different curves (from the top down) are 100, 10, 2, and 1 ps.
For $j \geq j_{\text {th2 }}$, the current density of stimulated recombination via the ground-state in QDs is pinned [see Eq. (52)]. Hence the internal efficiency for ground-state lasing simply decreases as the reciprocal of $j-j_{\text {th1 }}$

$$
\eta_{\text {int } 1}=\frac{j_{\text {stim } 1}^{\text {pin }}}{j-j_{\text {th } 1}} .
$$

Above $j_{\text {th2 }}$, the internal efficiency for excited-state lasing is

$$
\eta_{\text {int2 }}=\frac{j_{\text {stim2 }}}{j-j_{\text {th2 }}} .
$$

With (C5) for $j_{\text {stim2 }}(j)$, the following expression is obtained for $\eta_{\text {int2 }}$ :

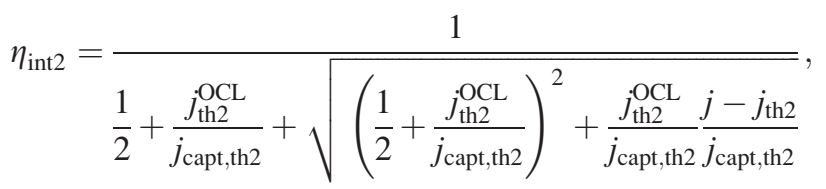

which is similar to Eq. (31) of Ref. 22.

Fig. 11 shows the internal differential quantum efficiency against injection current density.

Above $j_{\text {th } 2}, P_{1}$ is pinned at $P_{1}^{\text {pin }}$. Hence, the external differential quantum efficiency is given by the derivative of only $P_{2}$ with respect to $j$

$$
\eta_{\text {ext } 2}=\frac{1}{\frac{\hbar \omega_{2}}{e}} \frac{1}{S} \frac{\partial P_{2}}{\partial j}=\frac{\partial j_{\mathrm{stim} 2}}{\partial j} .
$$

With Eq. (48), we have

$$
\begin{aligned}
& \eta_{\mathrm{ext} 2}=\frac{1}{\sqrt{\left(1+2 \frac{j_{\mathrm{th} 2}^{\mathrm{OCL}}}{j_{\mathrm{capt}, \mathrm{th} 2}}\right)^{2}+4 \frac{j_{\mathrm{th} 2}^{\mathrm{OCL}}}{j_{\mathrm{capt}, \mathrm{th} 2}} \frac{j-j_{\mathrm{th} 2}}{j_{\mathrm{capt}, \mathrm{th} 2}}}} . \\
&=\frac{1}{\sqrt{1+4 \frac{j_{\mathrm{th} 2}^{\mathrm{OCL}}}{j_{\text {capt } \text { th2 }}^{2}}\left(j_{\mathrm{esc} 2}^{\mathrm{OCL}}+j\right)}} .
\end{aligned}
$$

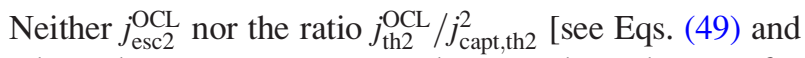
(50)] depends on $\tau_{21}$. Hence $\eta_{\text {ext2 }}$ does not depend on $\tau_{21}$ for $j \geq j_{\text {th2 }}$; this is also seen from Fig. 12-the curves for $\eta_{\text {ext2 }}$ calculated at different values of $\tau_{21}$ merge together above $j_{\text {th } 2}$.

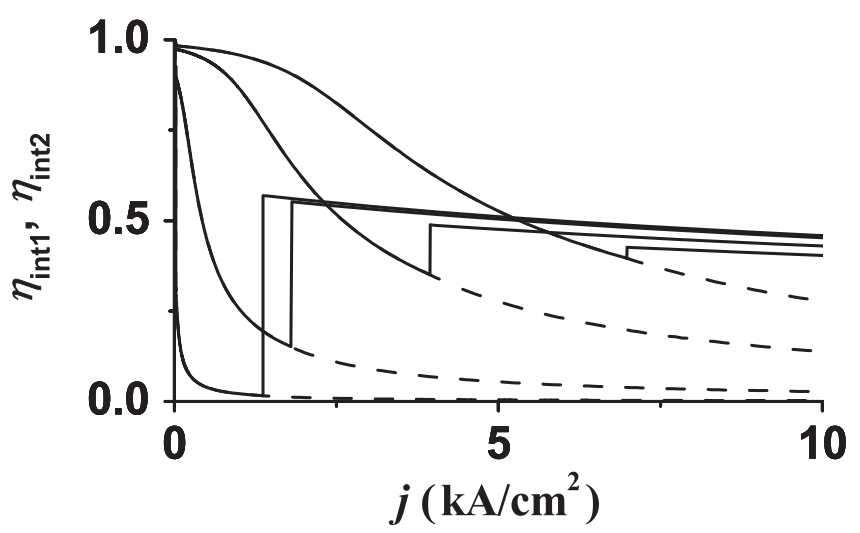

FIG. 11. Internal differential quantum efficiency against injection current density. The values of $\tau_{21}$ for different curves (from left to right) are 100, 10,2 , and 1 ps. The solid curves show $\eta_{\text {int } 1}$ for $j \leq j_{\text {th2 }}$ and $\eta_{\text {int } 2}$ for $j>j_{\text {th2 }}$. The dashed curves show $\eta_{\text {int } 1}$ for $j \geq j_{\text {th2 } 2}$. 


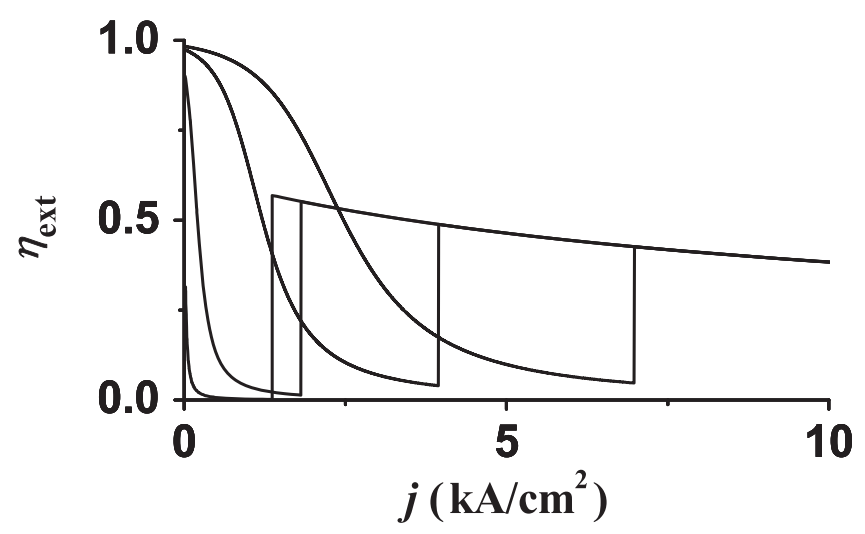

FIG. 12. External differential quantum efficiency against injection current density. The values of $\tau_{21}$ for different curves (from left to right) are 100, 10,2 , and $1 \mathrm{ps}$. The steps in the curves occur at $j=j_{\mathrm{th} 2}$.

At $j=j_{\text {th2 }}$, we have from Eqs. (57) and (59)

$$
\eta_{\text {int }, \text { th2 }}=\eta_{\text {ext }, \text { th2 }}=\frac{1}{1+2 \frac{j_{\text {th2 }}^{\text {oCL }}}{j_{\mathrm{capt} \text { th2 }}}} .
$$

In the absence of piezoelectric effects (which is not always the case-see, e.g., Refs. 32 and 33), transitions from excited electron- to excited hole-states are degenerate in pyramidal (with square base), cubic, and cylindrical QDs; hence, the maximum gain for excited-state transitions $g_{2}^{\max }$ could be higher than that for ground-state transitions $g_{1}^{\max }$. If such would be the case, the findings of Section III should be modified as follows. If the mirror reflectivity at the groundand excited-state transition energies is the same $\left(R_{1}=R_{2}\right.$ and hence $\beta_{1}=\beta_{2}$ ) and both $g_{1}^{\max }$ and $g_{2}^{\max }$ are higher than the mirror loss, excited-state lasing will turn on first with increasing pump current and then so will do ground-state lasing. If $R_{1} \neq R_{2}$ and $g_{1}^{\max }$ and $g_{2}^{\max }$ are higher than the mirror losses $\beta_{1}$ and $\beta_{2}$, respectively, whether lasing will first occur via ground- or excited-state transitions will be determined by the lower of the two threshold currents. If $j_{\text {th } 1}<j_{\text {th2 }}$, Section III remains unchanged. If $j_{\text {th } 2}<j_{\text {th } 1}$, Section III will still apply if the terms "ground" and "excited" and all the related quantities are interchanged throughout the text and expressions.

\section{EXCITED-STATE LASING ONLY: LOW GAIN FOR GROUND-STATE TRANSITIONS AND HIGH GAIN FOR EXCITED-STATE TRANSITIONS}

In Section III, we considered the situation when inequality Eq. (40) is satisfied. If the reverse condition holds, there will be excited-state lasing only. Qualitatively, this situation is similar to that for single-state QDs considered in Refs. 22 and 23.

Dropping out the stimulated recombination term in Eq. (3), the rate equation for the QD ground-state occupancy reads as

$$
\frac{f_{2}\left(1-f_{1}\right)}{\tau_{21}}-\frac{f_{1}\left(1-f_{2}\right)}{\tau_{12}}-\frac{f_{1}^{2}}{\tau_{\mathrm{QD} 1}}=0 .
$$

The rate equations for the QD excited-state occupancy, free-carrier density in the OCL, and photon density are given by Eqs. (41), (1), and (34), respectively.

\section{A. Solutions of rate equations}

At and above the excited-state lasing threshold, the excited-state occupancy $f_{2}$ is pinned at its threshold value $f_{2, \text { th2 }}$ given by Eq. (35). From Eq. (61) (which is a quadratic equation in $f_{1}$ ), we find that the ground-state occupancy $f_{1}$ is also pinned at the following value:

$$
f_{1, \mathrm{th} 2}=\frac{f_{2, \mathrm{th} 2}}{\frac{1}{2}\left[f_{2, \mathrm{th} 2}+\frac{\tau_{21}}{\tau_{12}}\left(1-f_{2, \mathrm{th} 2}\right)\right]+\sqrt{\frac{1}{4}\left[f_{2, \mathrm{th} 2}+\frac{\tau_{21}}{\tau_{12}}\left(1-f_{2, \mathrm{th} 2}\right)\right]^{2}+\frac{\tau_{21}}{\tau_{\mathrm{QD} 1}} f_{2, \mathrm{th} 2}}} .
$$

With Eq. (61), we have from Eq. (41)

$$
\begin{aligned}
n_{\mathrm{OCL}}= & n_{2} \frac{f_{2, \mathrm{th} 2}}{1-f_{2, \mathrm{th} 2}} \\
& +\frac{1}{\sigma_{2} \mathrm{v}_{\mathrm{n}}} \frac{1}{1-f_{2, \mathrm{th} 2}}\left(\frac{f_{1, \mathrm{th} 2}^{2}}{\tau_{\mathrm{QD} 1}}+\frac{f_{2, \mathrm{th} 2}^{2}}{\tau_{\mathrm{QD} 2}}+\frac{b}{N_{\mathrm{S}}} \frac{n_{\mathrm{ph} 2}}{\tau_{\mathrm{ph} 2}}\right) .
\end{aligned}
$$

With Eqs. (41) and (61), we have from Eq. (1)

$$
j=e b B n_{\mathrm{OCL}}^{2}+e N_{\mathrm{S}} \frac{f_{1, \mathrm{th} 2}^{2}}{\tau_{\mathrm{QD} 1}}+e N_{\mathrm{S}} \frac{f_{2, \mathrm{th} 2}^{2}}{\tau_{\mathrm{QD} 2}}+e b \frac{n_{\mathrm{ph} 2}}{\tau_{\mathrm{ph} 2}},
$$

where $n_{\mathrm{OCL}}$ is given by Eq. (63).

At the excited-state lasing threshold (when $n_{\mathrm{ph} 2}=0$ ), we have from Eqs. (63) and (64)

$$
\begin{gathered}
n_{\mathrm{OCL}, \mathrm{th} 2}=n_{2} \frac{f_{2, \mathrm{th} 2}}{1-f_{2, \mathrm{th} 2}}+\frac{1}{\sigma_{2} \mathrm{v}_{\mathrm{n}}} \frac{1}{1-f_{2, \mathrm{th} 2}}\left(\frac{f_{1, \mathrm{th} 2}^{2}}{\tau_{\mathrm{QD} 1}}+\frac{f_{2, \mathrm{th} 2}^{2}}{\tau_{\mathrm{QD} 2}}\right), \\
j_{\mathrm{th} 2}=e b B n_{\mathrm{OCL}, \mathrm{th} 2}^{2}+e N_{\mathrm{S}} \frac{f_{1, \mathrm{th} 2}^{2}}{\tau_{\mathrm{QD} 1}}+e N_{\mathrm{S}} \frac{f_{2, \mathrm{th} 2}^{2}}{\tau_{\mathrm{QD} 2}} .
\end{gathered}
$$

Eqs. (63)-(66) could also be immediately obtained from Eqs. (42) and (44)-(46) by putting there $n_{\mathrm{ph} 1}^{\mathrm{pin}}=0$ and $f_{1}=f_{1 \text {,th2 }}$.

With Eqs. (47) and (49) for $j_{\text {stim2 }}$ and $j_{\text {capt,th2, Eqs. (63) }}$ and (64) can be rewritten in the form of Eqs. (C2) and (C3) (see Appendix C). Consequently, the stimulated recombination current density $j_{\text {stim2 }}$, photon density $n_{\mathrm{ph} 2}$, output power $P_{2}$, internal and external efficiencies $\eta_{\text {int2 }}$ and $\eta_{\text {ext2 }}$ will be given as functions of the injection current density by Eqs. (C5), (48), (57), and (59). 


\section{CONCLUSIONS}

We developed a theory of operating characteristics of QD lasers in the presence of excited states in QDs. We considered three possible situations for lasing.

Under the conditions of ground-state lasing only (high gain for ground-state transitions and low gain for excitedstate transitions), the output power asymptotically approaches its maximum (saturation) value with increasing injection current. A simple universal expression is obtained for the normalized maximum power as a function of the normalized intradot relaxation time.

Under the conditions of both ground- and excited-state lasing, the output power of ground-state lasing remains pinned above the excited-state lasing threshold while the power of excited-state lasing increases. At the excited-state lasing threshold, a kink appears in the LCC. Above the excited-state lasing threshold, the free carrier density in the OCL, current of total stimulated recombination via the ground and excited states in QDs, and external differential quantum efficiency become independent of the intradot relaxation time. As in the case of ground-state lasing only, there exists a cut-off value of the intradot relaxation time, at which the output power of ground-state lasing vanishes. With increasing relaxation time, the threshold current for ground-state lasing increases while that for excited-state lasing decreases. At the cut-off value of the relaxation time, the threshold currents for ground- and excited-state lasing become the same.

The case of excited-state lasing only (low gain for ground-state transitions and high gain for excited-state transitions) is qualitatively similar to that for single-state QDs. Above the lasing threshold (which is the excited-state lasing threshold in this case), the role of ground-state transitions is simply reduced to adding an extra component (current of spontaneous recombination via the ground-state in QDs) to the threshold current. The free-carrier density in the OCL is also increased due to spontaneous recombination via the ground-state in QDs.

\section{ACKNOWLEDGMENTS}

This work was supported by the U.S. Army Research Office (Grant No. W911NF-13-1-0445). Y.W. also acknowledges the Fundamental Research Funds for the Central Universities (Grant No. 2015QNA10) for support of this work.

\section{APPENDIX A: GROUND-STATE LASING ONLY: LCC AT HIGH INJECTION CURRENTS} Eq. (25)

With Eqs. (8) and (22) for $\tau_{\mathrm{ph} 1}$ and $\tau_{21}^{\max }$, we have from

$$
\frac{b}{N_{\mathrm{S}}} \frac{1}{\tau_{\mathrm{ph} 1}}=\frac{\frac{1}{\tau_{21}}-\frac{1}{\tau_{21}^{\max }}}{n_{\mathrm{ph} 1}^{\max }}\left(1-f_{1}\right) .
$$

With Eqs. (A1) and (22), Eq. (7) can be written as

$$
1-f_{2}=\frac{\left(1-f_{1}\right)\left(1-\frac{\tau_{21}}{\tau_{21}^{\max }}\right)\left(1-\frac{n_{\mathrm{ph} 1}}{n_{\mathrm{ph} 1}^{\max }}\right)}{1-f_{1}+\frac{\tau_{21}}{\tau_{12}} f_{1}} .
$$

Using Eq. (A2) for $1-f_{2}$ in the denominators in Eq. (10), we have

$$
\begin{aligned}
n_{\mathrm{OCL}}= & \left\{n_{2} f_{2}+\frac{1}{\sigma_{2} \mathrm{v}_{\mathrm{n}}}\left[\frac{f_{2}\left(1-f_{1}\right)}{\tau_{21}}-\frac{f_{1}\left(1-f_{2}\right)}{\tau_{12}}+\frac{f_{2}^{2}}{\tau_{\mathrm{QD} 2}}\right]\right\} \\
& \times \frac{1-f_{1}+\frac{\tau_{21}}{\tau_{12}} f_{1}}{\left(1-f_{1}\right)\left(1-\frac{\tau_{21}}{\tau_{21}^{\max }}\right)} \frac{1}{1-\frac{n_{\mathrm{ph} 1}}{n_{\mathrm{ph} 1}^{\max }}} .
\end{aligned}
$$

As $n_{\mathrm{ph} 1}$ tends to $n_{\mathrm{ph} 1}^{\max }$ (i.e., as $f_{2} \rightarrow 1$ ), the free carrier density $n_{\mathrm{OCL}}$ in the OCL tends to infinity. The asymptotic expression for $n_{\mathrm{OCL}}$ is apparent from Eq. (A3)

$$
\begin{aligned}
n_{\mathrm{OCL}}= & {\left[n_{2}+\frac{1}{\sigma_{2} \mathrm{~V}_{\mathrm{n}}}\left(\frac{1-f_{1}}{\tau_{21}}+\frac{1}{\tau_{\mathrm{QD} 2}}\right)\right] } \\
& \times \frac{1-f_{1}+\frac{\tau_{21}}{\tau_{12}} f_{1}}{\left(1-f_{1}\right)\left(1-\frac{\tau_{21}}{\tau_{21}^{\max }}\right)} \frac{1}{1-\frac{n_{\mathrm{ph} 1}}{n_{\mathrm{ph} 1}^{\max }} \propto \frac{1}{1-\frac{n_{\mathrm{ph} 1}}{n_{\mathrm{ph} 1}^{\max }}}} .
\end{aligned}
$$

At high $n_{\mathrm{OCL}}$, the first term in the right-hand side of Eq. (12) becomes dominant. Thus Eq. (12) becomes

$$
j=e b B n_{\mathrm{OCL}}^{2} \propto \frac{1}{\left(1-\frac{n_{\mathrm{ph} 1}}{n_{\mathrm{ph} 1}^{\max }}\right)^{2}} .
$$

With Eqs. (A4) and (A5), we obtain the following asymptotic expression for the LCC at high $j$ :

$$
\begin{aligned}
\frac{P_{1}}{P_{1}^{\max }}= & \frac{n_{\mathrm{ph} 1}}{n_{\mathrm{ph} 1}^{\max }}=1-\frac{1-f_{1}+\frac{\tau_{21}}{\tau_{12}} f_{1}}{\left(1-f_{1}\right)\left(1-\frac{\tau_{21}}{\tau_{21}^{\max }}\right)} \\
& \times\left[n_{2}+\frac{1}{\sigma_{2} \mathrm{v}_{\mathrm{n}}}\left(\frac{1}{\tau_{\mathrm{QD} 2}}+\frac{1-f_{1}}{\tau_{21}}\right)\right] \sqrt{\frac{e b B}{j}}=1-\frac{\text { const }}{\sqrt{j}} .
\end{aligned}
$$

The asymptotic expression for the free carrier density in the OCL versus $j$ is apparent from Eq. (A5)

$$
n_{\mathrm{OCL}}=\sqrt{\frac{j}{e b B}} .
$$

At high current density (when $n_{\mathrm{ph} 1} \rightarrow n_{\mathrm{ph} 1}^{\max }$ ), the asymptotic expression for $\eta_{\mathrm{int} 1}$ is easily obtained from Eq. (27)

$$
\eta_{\text {int } 1}=\frac{j_{\text {stim } 1}^{\max }}{j}=\frac{e b \frac{n_{\mathrm{ph} 1}^{\max }}{\tau_{\mathrm{ph} 1}}}{j},
$$


where we introduced the saturation value $j_{\mathrm{stim} 1}^{\max }$ of the current density of stimulated recombination via the ground-state in QDs.

With Eq. (30) and the asymptotic expression Eq. (A6) for $P_{1}$, we have for the asymptotic expression for $\eta_{\operatorname{ext} 1}$ at high $j$

$$
\begin{aligned}
\eta_{\mathrm{ext} 1}= & \frac{1}{2} \frac{1-f_{1}+\frac{\tau_{21}}{\tau_{12}} f_{1}}{\left(1-f_{1}\right)\left(1-\frac{\tau_{21}}{\tau_{21}^{\max }}\right)} \\
& \times\left[n_{2}+\frac{1}{\sigma_{2} \mathrm{v}_{\mathrm{n}}}\left(\frac{1}{\tau_{\mathrm{QD} 2}}+\frac{1-f_{1}}{\tau_{21}}\right)\right] j_{\mathrm{stim} 1}^{\max } \\
& \times \frac{\sqrt{e b B}}{j^{3 / 2}} \propto \frac{1}{j^{3 / 2}} .
\end{aligned}
$$

\section{APPENDIX B: GROUND-STATE LASING ONLY: INSTANTANEOUS INTRADOT RELAXATION}

In the limiting case of instantaneous intradot relaxation $\left(\tau_{21}=0\right)$, Eqs. (7), (11), and (13) become

$$
\begin{aligned}
& f_{2}=\frac{f_{1}}{f_{1}+\left(1-f_{1}\right) \exp \left(\frac{\Delta}{T}\right)}, \\
& n_{\mathrm{OCL}}=n_{2} \frac{f_{1}}{1-f_{1}} \exp \left(-\frac{\Delta}{T}\right)+\frac{1}{\sigma_{2} \mathrm{v}_{\mathrm{n}}} \frac{1}{1-f_{1}} \\
& \times \exp \left(-\frac{\Delta}{T}\right)\left[f_{1}+\left(1-f_{1}\right) \exp \left(\frac{\Delta}{T}\right)\right] \\
& \times\left(f_{1}^{2}\left\{\frac{1}{\tau_{\mathrm{QD} 1}}+\frac{1}{\tau_{\mathrm{QD} 2}} \frac{1}{\left[f_{1}+\left(1-f_{1}\right) \exp \left(\frac{\Delta}{T}\right)\right]^{2}}\right\}\right. \\
& \left.+\frac{b}{N_{\mathrm{S}}} \frac{n_{\mathrm{ph} 1}}{\tau_{\mathrm{ph} 1}}\right) \\
& j=e b B n_{\mathrm{OCL}}^{2}+e N_{\mathrm{S}} f_{1}^{2} \\
& \times\left\{\frac{1}{\tau_{\mathrm{QD} 1}}+\frac{1}{\tau_{\mathrm{QD} 2}} \frac{1}{\left[f_{1}+\left(1-f_{1}\right) \exp \left(\frac{\Delta}{T}\right)\right]^{2}}\right\}+e b \frac{n_{\mathrm{ph} 1}}{\tau_{\mathrm{ph} 1}} .
\end{aligned}
$$

In Eqs. (B1)-(B3), Eq. (9) was used.

\section{APPENDIX C: GROUND- AND EXCITED-STATE LASING: CURRENT DENSITY OF STIMULATED RECOMBINATION VIA THE EXCITED-STATE IN QDS}

With Eq. (45), we can write Eq. (42) for the free-carrier density in the OCL as

$$
n_{\mathrm{OCL}}=n_{\mathrm{OCL}, \mathrm{th} 2}+\frac{1}{\sigma_{2} \mathrm{v}_{\mathrm{n}}} \frac{1}{1-f_{2, \mathrm{th} 2}} \frac{b}{N_{\mathrm{S}}} \frac{n_{\mathrm{ph} 2}}{\tau_{\mathrm{ph} 2}},
$$

or, using Eqs. (47) and (49), as

$$
n_{\mathrm{OCL}}=n_{\mathrm{OCL}, \mathrm{th} 2}\left(1+\frac{j_{\mathrm{stim} 2}}{j_{\mathrm{capt}, \mathrm{th} 2}}\right) .
$$

With Eqs. (46) and (47), we can rewrite Eq. (44) as

$$
j=j_{\text {th } 2}+e b B\left(n_{\mathrm{OCL}}^{2}-n_{\mathrm{OCL}, \mathrm{th} 2}^{2}\right)+j_{\mathrm{stim} 2} .
$$

Substituting $n_{\mathrm{OCL}}$ from Eq. (C2) into Eq. (C3), we have the following quadratic equation in $j_{\text {stim2 }}$ :

$$
\frac{j-j_{\text {th2 }}}{j_{\text {th2 }}^{\mathrm{OCL}}}=\left(1+\frac{j_{\text {stim2 }}}{j_{\text {capt } \text { th2 } 2}}\right)^{2}-1+\frac{j_{\text {stim2 }}}{j_{\text {th2 }}^{\mathrm{OCL}}} .
$$

The solution of this equation is

$$
\begin{aligned}
j_{\mathrm{stim} 2}= & j_{\text {capt } \mathrm{th} 2} \\
& \times\left[\sqrt{\left(1+\frac{1 j_{\text {capt }, \mathrm{th} 2}^{2}}{j_{\mathrm{th} 2}^{\mathrm{OCL}}}\right)^{2}+\frac{j-j_{\mathrm{th} 2}}{j_{\mathrm{th} 2}^{\mathrm{OCL}}}}-\left(1+\frac{1 j_{\text {capt } \mathrm{th} 2}}{j_{\mathrm{th} 2}^{\mathrm{OCL}}}\right)\right] .
\end{aligned}
$$

With Eq. (C5), we have for the free-carrier density from Eq. (C2)

$n_{\mathrm{OCL}}=n_{\mathrm{OCL}, \mathrm{th} 2}\left[\sqrt{\left(1+\frac{1}{2} \frac{j_{\mathrm{capt}, \mathrm{th} 2}}{j_{\mathrm{th} 2}^{\mathrm{OCL}}}\right)^{2}+\frac{j-j_{\mathrm{th} 2}}{j_{\mathrm{th} 2}^{\mathrm{OCL}}}}-\frac{1}{2} \frac{j_{\text {capt }, \mathrm{th} 2}}{j_{\mathrm{th} 2}^{\mathrm{OCL}}}\right]$.

${ }^{1}$ D. Arsenijević, A. Schliwa, H. Schmeckebier, M. Stubenrauch, M. Spiegelberg, D. Bimberg, V. Mikhelashvili, and G. Eisenstein, "Comparison of dynamic properties of ground- and excited-state emission in p-doped InAs/GaAs quantum-dot lasers," Appl. Phys. Lett. 104(18), 181101 (2014).

${ }^{2}$ C. Wang, B. Lingnau, K. Lüdge, J. Even, and F. Grillot, "Enhanced dynamic performance of quantum dot semiconductor lasers operating on the excited state," IEEE J. Quantum Electron. 50(9), 723-731 (2014).

${ }^{3}$ Y. Kaptan, A. Röhm, B. Herzog, B. Lingnau, H. Schmeckebier, D. Arsenijević, V. Mikhelashvili, O. Schöps, M. Kolarczik, G. Eisenstein, D. Bimberg, U. Woggon, N. Owschimikow, and K. Lüdge, "Stability of quantum-dot excited-state laser emission under simultaneous ground-state perturbation," Appl. Phys. Lett. 105(19), 191105 (2014).

${ }^{4}$ V. V. Korenev, A. V. Savelyev, A. E. Zhukov, A. V. Omelchenko, and M. V. Maximov, "Effect of carrier dynamics and temperature on two-state lasing in semiconductor quantum dot lasers," Semiconductors 47(10), 1397-1404 (2013).

${ }^{5}$ C. Y. Liu, H. Wang, Q. Q. Meng, B. Gao, and K. S. Ang, "Modal gain and photoluminescence investigation of two-state lasing in GaAs-based 1.3 $\mu \mathrm{m}$ InAs/InGaAs quantum dot lasers," Appl. Phys. Express 6(10), 102702 (2013).

${ }^{6} \mathrm{M}$. Gioannini, "Ground-state power quenching in two-state lasing quantum dot lasers," J. Appl. Phys. 111(4), 043108 (2012).

${ }^{7}$ V. V. Korenev, A. V. Savelyev, A. E. Zhukov, A. V. Omelchenko, and M. V. Maximov, "Analytical approach to the multi-state lasing phenomenon in quantum dot lasers," Appl. Phys. Lett. 102(11), 112101 (2013).

${ }^{8}$ J. Lee and D. Lee, "Double-state lasing from semiconductor quantum dot laser diodes caused by slow carrier relaxation," J. Korean Phys. Soc. 5(2), 239-242 (2011).

${ }^{9} \mathrm{~K}$. Schuh, F. Jahnke, and M. Lorke, "Rapid adiabatic passage in quantum dots: Influence of scattering and dephasing," Appl. Phys. Lett. 99(1), 011105 (2011). 
${ }^{10}$ W. W. Chow, M. Lorke, and F. Jahnke, "Will quantum dots replace quantum wells as the active medium of choice in future semiconductor lasers?," IEEE J. Sel. Top. Quantum Electron. 17(5), 1349-1355 (2011).

${ }^{11}$ F. Grillot, N. A. Naderi, J. B. Wright, R. Raghunathan, M. T. Crowley, and L. F. Lester, "A dual-mode quantum dot laser operating in the excited state," Appl. Phys. Lett. 99(23), 231110 (2011).

${ }^{12}$ Q. Cao, S. F. Yoon, C. Z. Tong, C. Y. Ngo, C. Y. Liu, R. Wang, and H. X. Zhao, "Two-state competition in $1.3 \mu \mathrm{m}$ multilayer InAs/InGaAs quantum dot lasers," Appl. Phys. Lett. 95(19), 191101 (2009).

${ }^{13}$ G. A. P. Thé, M. Gioannini, and I. Montrosset, "Numerical analysis of the effects of carrier dynamics on the switch-on and gain-switching of quantum dot lasers," Opt. Quantum Electron. 40(14-15), 1111-1116 (2008).

${ }^{14}$ K. Veselinov, F. Grillot, P. Miska, E. Homeyer, P. Caroff, C. Platz, J. Even, X. Marie, O. Dehaese, S. Loualiche, and A. Ramdane, "Carrier dynamics and saturation effect in (113)B InAs/InP quantum dot lasers," Opt. Quantum Electron. 38(4-6), 369-379 (2006).

${ }^{15}$ L. Jiang and L. V. Asryan, "Excited-state-mediated capture of carriers into the ground state and the saturation of optical power in quantum-dot lasers," IEEE Photonics Technol. Lett. 18(24), 2611-2613 (2006).

${ }^{16}$ M. Sugawara, N. Hatori, H. Ebe, M. Ishida, Y. Arakawa, T. Akiyama, K. Otsubo, and Y. Nakata, "Modeling room-temperature lasing spectra of 1.3- $\mu \mathrm{m}$ self-assembled InAs/GaAs quantum-dot lasers: Homogeneous broadening of optical gain under current injection," J. Appl. Phys. 97(4), 043523 (2005).

${ }^{17}$ E. A. Viktorov, P. Mandel, Y. Tanguy, J. Houlihan, and G. Huyet, "Electron-hole asymmetry and two-state lasing in quantum dot lasers," Appl. Phys. Lett. 87(5), 053113 (2005)

${ }^{18}$ A. Markus, J. X. Chen, C. Paranthoën, A. Fiore, C. Platz, and O. GauthierLafaye, "Simultaneous two-state lasing in quantum-dot lasers," Appl. Phys. Lett. 82(12), 1818-1820 (2003).

${ }^{19}$ A. E. Zhukov, A. R. Kovsh, D. A. Livshits, V. M. Ustinov, and Zh. I. Alferov, "Output power and its limitation in ridge-waveguide $1.3 \mu \mathrm{m}$ wavelength quantum-dot lasers," Semicond. Sci. Technol. 18(8), 774-781 (2003).

${ }^{20}$ A. Markus, J. X. Chen, O. Gauthier-Lafaye, J. G. Provost, C. Paranthoën, and A. Fiore, "Impact of intraband relaxation on the performance of a quantum-dot laser," IEEE J. Sel. Top. Quantum Electron. 9(5), 1308-1314 (2003).

${ }^{21}$ P. Bhattacharya, S. Krishna, J. Phillips, P. J. McCann, and K. Namjou, "Carrier dynamics in self-organized quantum dots and their application to long-wavelength sources and detectors," J. Cryst. Growth 227-228, 27-35 (2001).
${ }^{22}$ L. V. Asryan, S. Luryi, and R. A. Suris, "Internal efficiency of semiconductor lasers with a quantum-confined active region," IEEE J. Quantum Electron. 39(3), 404-418 (2003).

${ }^{23}$ L. V. Asryan, S. Luryi, and R. A. Suris, "Intrinsic nonlinearity of the lightcurrent characteristic of semiconductor lasers with a quantum-confined active region," Appl. Phys. Lett. 81(12), 2154-2156 (2002).

${ }^{24}$ S. Grosse, J. H. H. Sandmann, G. von Plessen, J. Feldmann, H. Lipsanen, M. Sopanen, J. Tulkki, and J. Ahopelto, "Carrier relaxation dynamics in quantum dots-Scattering mechanisms and state-filling effects," Phys. Rev. B 55(7), 4473-4476 (1997).

${ }^{25}$ D. Gammon, E. S. Snow, B. V. Shanabrook, D. S. Katzer, and D. Park, "Homogeneous linewidths in the optical spectrum of a single gallium arsenide quantum dot," Science 273(5271), 87-90 (1996).

${ }^{26}$ J. H. H. Sandmann, S. Grosse, G. von Plessen, J. Feldmann, G. Hayes, R. Phillips, H. Lipsanen, M. Sopanen, and J. Ahopelto, "Carrier relaxation in (GaIn)As quantum dots,” Phys. Status Solidi A 164(1), 421-425 (1997).

${ }^{27}$ J. Singh, "Possibility of room temperature intra-band lasing in quantum dot structures," IEEE Photonics Technol. Lett. 8(4), 488-490 (1996).

${ }^{28}$ D. Klotzkin, K. Kamath, and P. Bhattacharya, "Quantum capture times at room temperature in high-speed $\mathrm{In}_{0.4} \mathrm{Ga}_{0.6} \mathrm{As}-\mathrm{GaAs}$ self-organized quantum-dot lasers," IEEE Photonics Technol. Lett. 9(10), 1301-1303 (1997).

${ }^{29}$ I. V. Ignatiev, I. E. Kozin, S. V. Nair, H. W. Ren, S. Sugou, and Y. Masumoto, "Carrier relaxation dynamics in InP quantum dots studied by artificial control of nonradiative losses," Phys. Rev. B 61(23), 15633-15636 (2000).

${ }^{30}$ M. Grundmann, "How a quantum-dot laser turns on," Appl. Phys. Lett. 77(10), 1428-1430 (2000).

${ }^{31}$ P. Boucaud, K. S. Gill, J. B. Williams, M. S. Sherwin, W. V. Schoenfeld, and P. M. Petroff, "Saturation of THz-frequency intraband absorption in InAs/ GaAs quantum dot molecules,” Appl. Phys. Lett. 77(4), 510-512 (2000).

${ }^{32}$ L. V. Asryan, M. Grundmann, N. N. Ledentsov, O. Stier, R. A. Suris, and D. Bimberg, "Effect of excited-state transitions on the threshold characteristics of a quantum dot laser," IEEE J. Quantum Electron. 37(3), 418-425 (2001).

${ }^{33}$ L. V. Asryan, M. Grundmann, N. N. Ledentsov, O. Stier, R. A. Suris, and D. Bimberg, "Maximum modal gain of a self-assembled InAs/GaAs quantum-dot laser,” J. Appl. Phys. 90(3), 1666-1668 (2001).

${ }^{34}$ L. V. Asryan and R. A. Suris, "Inhomogeneous line broadening and the threshold current density of a semiconductor quantum dot laser," Semicond. Sci. Technol. 11(4), 554-567 (1996).

${ }^{35}$ L. V. Asryan and R. A. Suris, "Spatial hole burning and multimode generation threshold in quantum-dot lasers," Appl. Phys. Lett. 74(9), 1215-1217 (1999). 